\title{
GROTHENDIECK GROUPS OF QUOTIENT SINGULARITIES
}

\author{
EDUARDO DO NASCIMENTO MARCOS
}

\begin{abstract}
Given a quotient singularity $R=S^{G}$ where $S=\mathbf{C}\left[\left[x_{1}, \ldots, x_{n}\right]\right]$ is the formal power series ring in $n$-variables over the complex numbers $\mathbf{C}$, there is an epimorphism of Grothendieck groups $\psi: G_{0}(S[G]) \rightarrow G_{0}(R)$, where $S[G]$ is the skew group ring and $\psi$ is induced by the fixed point functor.

The Grothendieck group of $S[G]$ carries a natural structure of a ring, isomorphic to $G_{0}(\mathbf{C}[G])$.

We show how the structure of $G_{0}(R)$ is related to the structure of the ramification locus of $V$ over $V / G$, and the action of $G$ on it.

The first connection is given by showing that $\operatorname{Ker} \psi$ is the ideal generated by [C] if and only if $G$ acts freely on $V$. That this is sufficient has been proved by Auslander and Reiten in [4]. To prove the necessity we show the following:

Let $U$ be an integrally closed domain and $T$ the integral closure of $U$ in a finite Galois extension of the field of quotients of $U$ with Galois group $G$. Suppose that $|G|$ is invertible in $U$, the inclusion of $U$ in $T$ is unramified at height one prime ideals and $T$ is regular. Then $G_{0}(T[G]) \cong Z$ if and only if $U$ is regular. 0 .

We analyze the situation $V=V_{1} \amalg_{\mathbf{C}[G]} V_{2}$ where $G$ acts freely on $V_{1}, V_{1} \neq$

We prove that for a quotient singularity $R, G_{0}(R) \cong G_{0}(R[[t]])$.

We also study the structure of $G_{0}(R)$ for some cases with $\operatorname{dim} R=3$.
\end{abstract}

\section{INTRODUCTION}

The objective of this paper is to study connections between the structure of the Grothendieck group $G_{0}(R)$ of a quotient singularity $R=S^{G}$ and the ramification theory of the extension $R \rightarrow S$.

We begin by defining these concepts.

(1) We recall that a quotient singularity $R$ is a ring of the form $R=S^{G}$ where $S=\mathbf{C}\left[\left[x_{1}, \ldots, x_{n}\right]\right]$ is the formal power series in $n$ variables over the complex numbers $\mathrm{C}$ and $G$ is a finite subgroup of $G L(N, \mathrm{C})$. The latter group acts on $S$ by those $\mathbf{C}$-algebra automorphisms which are induced by the linear action on the variables. We assume always $n \geq 2$.

We remark that $R$ and $S$ are integrally closed noetherian complete local domains and that $S$ is a finitely generated module over $R$.

Every ring we consider is noetherian with one and every module is finitely generated.

(2) The inclusion of $G$ in $G L(n, \mathbf{C})$ gives an action of $G$ on the affine space $C^{n}$ so that we have a quotient variety $C^{n} / G$ and a surjective morphism

Received by the editors June 17, 1987 and, in revised form, March 1, 1990.

1980 Mathematics Subject Classification (1985 Revision). Primary 19A49. 
$\pi: \mathbf{C}^{n} \rightarrow \mathbf{C}^{n} / G$. In this situation we recall the following definitions:

(a) A point $y \in \mathbf{C}^{n}$ is called ramified if the cardinality of the orbit of $y$, $|G(y)|$, is smaller than $|G|$, the order of $G$.

(b) The ramification locus is the set of ramified points, i.e. $\left\{y \in \mathbf{C}^{n}: y\right.$ is ramified $\}$. This is a union of linear subspaces.

We suppose that $G$ contains no pseudo-reflections which means that all subspaces in the ramification locus have dimension less than $n-1$.

(c) Let $W$ be an irreducible subvariety of $\mathbf{C}^{n} / G$ and $I(W)$ its ideal in $\mathbf{C}\left[x_{1}, \ldots, x_{n}\right]^{G}$. Then $W$ is called unramified if there is a point in $W$ that is unramified. Since $R$ is the completion of $\mathbf{C}\left[x_{1}, \ldots, x_{n}\right]^{G}$ with respect to $\left(x_{1}, \ldots, x_{n}\right) \cap \mathbf{C}\left[x_{1}, \ldots, x_{n}\right]^{G}$ we have that if $\pi(0) \in W$ then $W$ is unramified if and only if $I(W) . R$ is unramified in $S$.

The hypothesis on the nonexistence of pseudo-reflections is equivalent to the assumption that the extension $R \rightarrow S$ is unramified at height one prime ideals.

(3) If 0 is the only ramified point of $\mathbf{C}^{n}$ we say that $G$ acts freely. This is equivalent to saying that $R_{\underline{p}}$ is regular for all $\underline{p}$ nonmaximal. A ring with this property is called an isolated singularity.

(4) We recall now the definition of the Grothendieck group, $G_{0}(\Lambda)$, of a ring $\Lambda$. It is the quotient of the free abelian group whose generators are the isomorphism classes of left modules $[M]$, with relations given by $[M]-\left[M^{\prime}\right]-$ $\left[M^{\prime \prime}\right]$ for each exact sequence $0 \rightarrow M^{\prime} \rightarrow M \rightarrow M^{\prime \prime} \rightarrow 0$ of finitely generated left $\Lambda$-modules.

We want to investigate how the structure of $G_{0}(R)$ is connected with the structure of the ramification locus and the action of $G$ on it.

We study the Grothendieck group $G_{0}(R)$ in terms of the Grothendieck group, $G_{0}(S[G])$, of the skew group ring $S[G]$.

The skew group ring $S[G]$ is the free module over $S$ with basis the elements of $G$ and the ring multiplication given by $\left(s_{1} \sigma_{1}\right)\left(s_{2} \sigma_{2}\right)=s_{1}\left(\sigma_{1}\left(s_{2}\right)\right)\left(\sigma_{1} \sigma_{2}\right)$ for $s_{1}, s_{2} \in S$ and $\sigma_{1}, \sigma_{2} \in G$.

An $S[G]$ module is an $S$-module with an action of $G$ s.t. $\sigma(s m)=\sigma(s) \sigma(m)$ for $\sigma \in G, s \in S$, and $m \in M$.

This study began with the work of Auslander and Reiten, Grothendieck groups of algebras and orders [4].

They defined an epimorphism $\psi: G_{0}(S[G]) \rightarrow G_{0}(R)$ by $[M] \rightarrow\left[M^{G}\right]$ for $M$ an $S[G]$-module, where $M^{G}=\{m \in M$. s.t. $\sigma(m)=m$, for all $\sigma \in G\}$.

Since $S[G]$ is regular and $S$ is complete one gets that $G_{0}(S[G])$ is the free abelian group generated by the isomorphism classes of indecomposable projective $S[G]$-modules which in turn are the classes of indecomposable direct summands of $S[G]$. Therefore $G_{0}(S[G])$ is a finitely generated free abelian group, the map $\psi$ gives a natural presentation of $G_{0}(R)$ and $G_{0}(R)$ is in particular finitely generated. Moreover, Auslander and Reiten proved that $G_{0}(R) \cong$ $Z[R] \amalg T$ where $T$ is finite.

If we take in $S[G]$ the idempotent $e=\sum_{\sigma \in G} \sigma /|G|$ and let $(\underline{e})$ denote the two-sided ideal of $S[G]$ generated by $e$ we have an exact sequence $G_{0}(S[G] /(\underline{e}))$ $\rightarrow G_{0}(S[G]) \stackrel{\psi}{\rightarrow} G_{0}(R) \rightarrow 0$.

We show that for $\underline{a}=\operatorname{Ker} \theta$, where $\theta$ is the ring homomorphism $\theta: S \rightarrow$ $S[G] /(\underline{e})$ given by $\theta(s)=\mathrm{s} .1$, a prime ideal $\underline{q}$ of $S$ is ramified over $R$ iff $\underline{q} \supset$ $\underline{a}$. We prove, in addition, that the natural map $G_{0}(S / \underline{a}[G] /(\underline{e})) \rightarrow G_{0}(S[G] /(\underline{e}))$ 
is an isomorphism. Hence we get an exact sequence

$$
G_{0}(S / \underline{a}[G] /(\underline{e})) \rightarrow G_{0}(S[G]) \stackrel{\psi}{\rightarrow} G_{0}(R) \rightarrow 0 .
$$

This yields a way of computing $\operatorname{Ker} \psi$. As the $\operatorname{ring}(S / \underline{a}[G] /(\underline{e}))$ is defined in terms of the ramification, it follows that there is a clear connection between $\operatorname{Ker} \psi$ and the ramification.

Since $\operatorname{dim} R \geq 1, R$ is complete and $R / \underline{m} R \cong \mathbf{C}$ is algebraically closed, it follows that the classes of finite length modules are zero in $G_{0}(R)$. As a consequence, the classes of finite length $S[G]$-modules are in $\operatorname{Ker} \psi$.

The subgroup of $G_{0}(S[G])$ generated by the classes of f.l. $S[G]$-modules can be described by putting the ring structure on $G_{0}(S[G])$ for which $\left[P_{1}\right] \cdot\left[P_{2}\right]=$ $\left[P_{1} \otimes_{S} P_{2}\right]$, if $P_{1}$ and $P_{2}$ are two projective $S[G]$-modules.

We remark that $G_{0}(S[G])$ is isomorphic to the classical ring of representations $G_{0}(\mathbf{C}[G])$.

The subgroup of $G_{0}(S[G])$ generated by the classes of finite length $S[G]$ modules is the principal ideal $[\mathbf{C}] G_{0}(S[G])$ where $\mathbf{C}=S / \underline{m}_{S}$ as an $S[G]$ module.

Since $R$ is integrally closed there is a natural epimorphism

$$
G_{0}(R) \stackrel{\pi}{\rightarrow} Z \amalg \mathrm{Cl}(R) \rightarrow 0,
$$

where $\mathrm{Cl}(R)$ is the divisor class group. In our case $\mathrm{Cl}(R)$ is isomorphic to $G^{*}=\operatorname{Hom}(G, \mathbf{C}-\{0\})$.

We show that the kernel of the composition $\pi \psi: G_{0}([G]) \rightarrow Z \amalg \mathrm{Cl}(R) \rightarrow 0$ is an ideal $\underline{J}$ that contains $\operatorname{Ker} \psi$. Moreover $\underline{I}^{2} \subset \underline{J} \subset \underline{I}$ where $\underline{I}=\operatorname{Ker} \varepsilon$ and $\varepsilon: G_{0}(S[G]) \rightarrow Z$ is the map $\varepsilon([M])=\operatorname{rank}_{S} M$ for $M$ any $S[G]$-module.

So we have $[\mathbf{C}] G_{0}(S[G]) \subset \operatorname{Ker} \psi \subset \underline{J}$.

In general $\operatorname{Ker} \psi$ is not an ideal and the examples we have, show that the property of $\operatorname{Ker} \psi$ being an ideal depends strongly on the action of $G$ on the ramification locus.

If $\operatorname{Ker} \psi$ is an ideal one can use $\psi$ to put a ring structure on $G_{0}(R)$.

We have the following characterization of $R$ being an isolated singularity.

$R$ is an isolated singularity $\Leftrightarrow \operatorname{Ker} \psi=[\mathbf{C}] G_{0}(S[G])$.

The implication $(\Rightarrow)$ is based on the following new characterization of regularity of a fixed point ring of a regular ring.

Let $U$ be an integrally closed local domain and $T$ the integral closure of $U$ in a finite Galois extension of the field of quotients of $U$ with Galois group $G$. Suppose that $|G|$ is invertible in $U$ and that $U \rightarrow T$ is unramified at height one prime ideals and that $T$ is regular. Then $\operatorname{rank} G_{0}(T[G])=1$ iff $U$ is regular.

We prove that, for a quotient singularity $R, G_{0}(R) \cong G_{0}(R[[t]])$ the isomorphism being given by the canonical mapping, $[M] \rightarrow[M[[t]]]$ for $M$ an $R$-module. We give a counterexample showing that this is not true for a more general ring $U$. We do not know a good characterization of rings $U$ s.t. $G_{0}(U) \cong G_{0}(U[[t]])$.

At the end we study some cases of three-dimensional quotient singularities. We deal mainly with the case of $G$ a cyclic group. There we give a description of $\operatorname{Ker} \psi$ and we describe some cases where $\operatorname{Ker} \psi$ is an ideal.

This paper consists almost entirely of my (1987) doctoral dissertation at Brandeis University. I would like to take this opportunity to thank Professor 
Maurice Auslander, my thesis advisor, for many suggestions, ideas and helpful discussions, I also thank Professor D. Farkas for helpful discussions and Professor E. Green for having made my stay at VPI \& SU for one year possible. Finally I thank CNPq and IME-USP, both of Brazil, for their support during these four years.

I want to thank the referee for his patience and Lynn Olinger for her patience in the typing.

\section{Chapter I}

Consider the epimorphism $\psi: G_{0}(S[G]) \rightarrow G_{0}(R)$. We know that the subgroup of $G_{0}(S[G])$ generated by the classes of finite length modules is the principal ideal $[\mathrm{C}] G_{0}(S[G])$; moreover we know that this subgroup is contained in $\operatorname{Ker} \psi$.

There is a map $\theta: G_{0}(R) \rightarrow Z \amalg \mathrm{Cl}(R) \rightarrow 0$ whose kernel is the subgroup of $G_{0}(R)$ generated by the classes $[R / \underline{p}]$ with $\underline{p}$ any prime ideal such that ht $p>2$.

Define on the group $Z \amalg \mathrm{Cl}(R)$ the ring structure whose identity is $(1,0)$ and whose multiplication is given by $(a, \alpha) \cdot(b, \beta)=(a b, a \beta+b \alpha)$ for all $a$, $b$ in $Z$ and $\alpha, \beta$ in $\mathrm{Cl}(R)$. It follows that for all pairs of reflexive $R$-modules $M$ and $N$ it holds that $\theta\left(M \otimes_{R} N\right)=\theta\left(\left(M \otimes_{R} N\right)^{* *}\right)=\theta(M) \cdot \theta(N)$. Therefore the group epimorphism $\theta \psi: G_{0}(S[G]) \rightarrow Z \amalg \mathrm{Cl}(R)$ satisfies

$$
\theta \psi[S]=(1,0)
$$

and

$$
\begin{aligned}
(\theta \psi)\left(\left[P_{1}\right] \cdot\left[P_{2}\right]\right) & =\theta \psi\left(\left[P_{1} \otimes_{S} P_{2}\right]\right)=\theta\left(\left[\left(P_{1} \otimes_{S} P_{2}\right)^{G}\right]\right) \\
& =\theta\left(\left(P_{1}^{G} \otimes_{R} P_{2}^{G}\right)^{* *}\right)=\theta \psi\left[P_{1}\right] \cdot \theta \psi\left[p_{2}\right] .
\end{aligned}
$$

Hence $\theta \psi$ is a ring map whose kernel $\underline{J}$ contains $\operatorname{Ker} \psi$. Moreover if we take $\varepsilon: G_{0}(S[G]) \rightarrow Z$ given by $\varepsilon([M])=\operatorname{rank}_{S}(M)$ and $\underline{I}=\operatorname{Ker} \varepsilon$ then $\underline{I}^{2} \subset \underline{J} \subset \underline{I}$ as for $x, y$ in $\underline{I}$ one has

$$
\theta \psi(x \cdot y)=\theta \psi(x) \cdot \theta \psi(y)=0 .
$$

If $G$ is commutative then $\left|I / I^{2}\right|=|G|=|\mathrm{Cl}(R)|$ and hence in this case $\underline{I}^{2}=\underline{J}$.

The remarks above show the following:

Proposition 1.1. Set

$$
\underline{J}=\operatorname{Ker}\left(G_{0}(S[G]) \rightarrow Z \amalg \mathrm{Cl}(R)\right) \quad \text { and } \quad \underline{I}=\operatorname{Ker}\left(G_{0}(S[G]) \rightarrow Z\right) .
$$

Then $\underline{J}$ is an ideal and $[\mathbf{C}] G_{0}(S[G]) \subset \operatorname{Ker} \psi \subset \underline{J}$. Moreover $\underline{I}^{2} \subset \underline{J} \subset \underline{I}$ and $\underline{I}^{2}=\underline{J}$ if $G$ is commutative.

We remark that if $\operatorname{Ker} \psi$ is an ideal then $G_{0}(R)$ inherits a natural ring structure. As we are going to show, whether $\operatorname{Ker} \psi$ is an ideal depends on how $G$ acts on the ramification locus.

We show that $\operatorname{Ker} \psi=G_{0}(S[G]) \cdot[C]$ iff $R$ is an isolated singularity, equivalently iff ramification locus $=\{0\}$.

We need for this some basic facts which we now recall. 
As a consequence of the purity of branch locus, see [1], we have the following: Suppose $U \subset T$ is an extension of normal local rings, with $T$ a finitely generated $U$-module. Suppose this extension is unramified at height one prime ideals and $T$ is regular. Then a prime $p$ of $U$ is unramified in $T$ if and only if $U_{p}$ is regular.

Moreover, see for example [4], we have the following: Suppose that $G$ is a finite group of automorphisms of a commutative ring $T$ with $T^{G}=U$. Then the following are equvialent:

(a) The fixed point functor $\underline{\underline{P}} T[G] \rightarrow \operatorname{add}_{U} T$, given by $P \rightarrow P^{G}$, from the category $\underline{\underline{P}} T[G]$ of projective $\overline{\bar{T}}[G]$-modules to the category $\operatorname{add}_{U} T$ of sums of direct summands of $T$, is an equivalence of categories.

(b) The $U$-algebra morphism $\gamma: T[G] \rightarrow \operatorname{End}_{U} T$ given by $\gamma(t \sigma)(x)=t \sigma(x)$ for all $t$ in $T, \sigma$ in $G$ and $x$ in $T$, is an isomorphism.

If in addition $T$ is normal, these conditions are also equivalent to:

(c) Each height one prime ideal $\underline{p}$ of $U$ is unramified in $T$.

We show now the following lemma:

Lemma 1.2. Suppose that $G$ is a finite group of automorphisms of a commutative ring $T$ with fixed point a ring $U=T^{G}, U$ a local ring. Let - denote completion. Then $G$ acts on $\widehat{T}$ by automorphisms. Moreover if the fixed point functors ()$^{G}: \underline{\underline{P}} T[G] \rightarrow \operatorname{add}_{U} T$ is an equivalence of categories then $\underline{\underline{P}} \widehat{T}[G] \rightarrow \operatorname{add}_{\widehat{U}} \widehat{T}$ is an equivalence of categories.

Proof. First we see that there is a natural action of $G$ on $\widehat{T}=\widehat{U} \otimes_{U} T$ by defining $\sigma(u \otimes t)=u \otimes \sigma(t)$ for $u \in \widehat{U}, t \in T, \sigma \in G$. It is easy to see that $\sigma\left(\lim _{n \rightarrow \infty} t_{n}\right)=\lim _{n \rightarrow \infty} \sigma\left(t_{n}\right)$ if $\left(t_{n}\right)_{n \in N}$ is a Cauchy sequence in $T$. If the map $\gamma: T[G] \rightarrow \operatorname{End}_{U} T, \gamma(t \sigma)(x)=t \sigma(x)$ is an isomorphism then $\hat{\gamma}: \widehat{T}[G] \rightarrow \operatorname{End}_{\widehat{U}} \widehat{T}$ is an isomorphism so ()$^{G}: \underline{\underline{P}} \widehat{T}[G] \rightarrow \operatorname{add}_{\widehat{U}} \widehat{T}$ is an equivalence.

We have as a consequence the following theorem:

Theorem 1.3. Let $U$ be a normal local ring with field of quotients $K$. Let $L$ be a finite Galois extension of $K$ with Galois group $G$ where $|G|$ is invertible in $U$, and $T$ the integral closure of $U$ in $L$. Suppose that $U \rightarrow T$ is unramified at height one prime ideals and that $T$ is regular. Then the following are equivalent.

(1) Up to isomorphism, there is a unique indecomposable module in $\underline{\underline{P}} T[G]$.

(2) $U$ is regular.

(3) $G_{0}(T[G]) \cong Z$.

(4) $\operatorname{rank} G_{0}(T[G])=1$.

Proof. (1) is equivalent to (2). Because $U \rightarrow T$ is unramified at height one primes we have that taking fixed points induces an equivalence of categories between $\underline{P} T[G]$ and $\operatorname{add}_{U} T$. Moreover $U$ is unramified in $T$ if and only if $U$ is regular and this happens if and only if $T$ is a projective $U$-module i.e. if and only if $\operatorname{add}_{U} T$ has only one indecomposable object up to isomorphism. Since $\operatorname{add}_{U} T$ is equivalent to $\underline{\underline{P}} T[G]$, we have the statement.

(1) implies (3).

Since $T$ is regular, $T[G]$ is regular and the Cartan map gives an isomorphism between $K_{0}(\underline{\underline{P}} T[G])$ and $G_{0}(T[G])$. So if there is only one class of indecomposable projective $T[G]$-modules then $G_{0}(T[G]) \cong Z$. 
(3) implies (4). This is obvious.

(4) implies (1):

Suppose first that $T$ is complete. Then the Krull-Schmidt Theorem holds for $T[G]$-modules. As $T[G]$ is also regular it follows that $G_{0}(T[G])$ is the free abelian group on the isomorphism classes of indecomposable projective $T[G]$-modules. So (4) implies (1) in this case.

Now suppose $T$ is not complete. Since $\hat{U}$ is flat over $U$ we have the canonical map

$$
\theta: G_{0}(T[G]) \rightarrow G_{0}(\widehat{T}[G]) \text { given by } \theta[M]=\left[\widehat{U} \otimes_{U} M\right]=[\widehat{M}] .
$$

$G_{0}(\widehat{T}[G])$ is the free abelian group in the classes of nonisomorphic indecomposable summands of $\widehat{T}[G]$. The equivalences of categories $\operatorname{add}_{\widehat{U}} \widehat{T}$ and $\underline{\underline{P}} \widehat{T}[G]$, given by the former lemma, shows that $\widehat{T}$ is an indecomposable $\widehat{T}[G]$ module, as it corresponds to $\widehat{U}$ under the equivalence. Since $[\widehat{T}[G]]=$ $\sum_{i=1}^{k} n_{i}\left[P_{i}\right]$ where $n_{i}$ is the multiplicity of each indecomposable $P_{i}$ in $\widehat{T}[G]$, it follows from $\operatorname{rank} G_{0}(T[G])=1$, that $[\widehat{T}]$ and $[\widehat{T}[G]]$ are linearly dependent in $G_{0}(\widehat{T}[G])$. Hence $k=1$ in that case and $\underline{\underline{P}} \widehat{T}[G]$ has only one indecomposable object, up to isomorphism, (namely $\widehat{T}$ ). Hence $\widehat{U} \rightarrow \widehat{T}$ is unramified and therefore $U \rightarrow T$ is unramified too. This implies that $\underline{\underline{P}} T[G]$ contains only one indecomposable object, up to isomorphism.

In this section we prove two theorems, the first of which establishes an exact diagram of Grothendieck groups, clarifying the strong connection between the structure of the group $\operatorname{Ker} \psi$ and the ramification theory of $R$ in $S$. Here $R=S^{G}, S=\mathbf{C}\left[\left[x_{1}, \ldots, x_{n}\right]\right]$ and $G$ is a finite subgroup of $G L(n, \mathbf{C})$. We assume throughout that $G$ has no pseudo-reflections, i.e. there is no nonidentity element $\sigma$ in $G$ s.t. $\operatorname{dim}_{\mathbf{C}}(\operatorname{Im}(1-\sigma)) \leq 1$. This is equivalent to the inclusion of $R$ in $S$ being unramified at height one prime ideals. As a consequence of the theorem on the purity of branch locus, [1], it follows that a prime ideal $\underline{p}$ in $R$ is unramified in $S$ if and only if $R_{\underline{p}}$ is regular.

Proposition 2.1. Let $G$ be a finite subgroup of $G L(n, \mathbf{C})$ without pseudo-reflections and $R=S^{G}$, where $S=\mathbf{C}\left[\left[x_{1}, \ldots, x_{n}\right]\right]$. Let $e=\frac{1}{|G|} \sum_{\sigma \in G} \sigma$ and $(\underline{e})$ the two-sided ideal generated by the idempotent e. Let $\underline{a}=\operatorname{Ker}(S \rightarrow S[G] /(\underline{e}))$ be given by $s \rightarrow \mathrm{s} .1$. Then a prime ideal $\underline{q}$ of $S$ is ramified $\Leftrightarrow \underline{a} \subset \underline{q}$. Moreover $\underline{a}$ and $\sqrt{a}$ are $G$-ideals.

Proof. Let $\underline{q} \in \operatorname{Spec} S$ and $\underline{p}=\underline{q} \cap R$. Then $(S[G] /(\underline{e})) \underline{p}=0 \Leftrightarrow\left(\underline{\operatorname{End}}_{R} S\right)_{p}=$ $0 \Leftrightarrow \underline{p}$ is unramified $\Leftrightarrow \exists \alpha \notin \underline{p}$ s.t. $\alpha S[G] \subset(\underline{e})$.

Thus if $\underline{q}$ is unramified there is $\alpha \in(e) \cap S$ with $\alpha \notin q$ s.t. $\alpha S[G] \subset(e)$ and $\underline{a} \supset \underline{q}$. If $\underline{a} \supset \underline{q}$ then $\left(\underline{\operatorname{End}}_{R} S\right)_{\underline{p}}=0$. This proves the first part of the statement.

For the second part, observe that if $\theta(a) \in(e)$ and $\sigma \in G$ then

$$
\theta(\alpha(a))=\sigma(a) \cdot 1=\sigma\left(\theta(a) \sigma^{-1}\right) \in(\underline{e}) .
$$


Now $s \in \sqrt{\underline{a}} \Leftrightarrow s^{k} \in \underline{a}$ for some $k \in N^{*}$. For such $k, \sigma(s)^{k}=\sigma\left(s^{k}\right) \in(\underline{a})$. Hence

$$
s \in \sqrt{\underline{a}} \Rightarrow \sigma(s) \in \sqrt{\underline{a}} \text { for all } \sigma \in G .
$$

We have the following corollary.

Corollary 2.2. Let $R \rightarrow S$, be unramified at height one prime ideals as before. Assume given a G-ideal $\underline{b}$ of $S$, i.e. an $S[G]-$ submodule of $S$, such that $\underline{b} \subset \sqrt{\underline{a}}$ where $\underline{a}=\operatorname{Ker}(S \rightarrow S[G] /(e))$. Then the following diagram is commutative and exact:

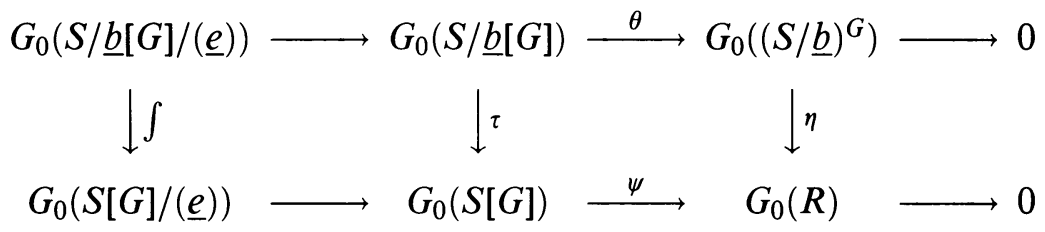

The maps $\psi$ and $\theta$ are induced by the functor of fixed points and all the others are induced by the canonical epimorphisms of rings.

Proof. It is easy to check that the diagram commutes. That the map

$$
G_{0}(S / \underline{b}[G] /(\underline{e})) \rightarrow G_{0}(S[G] /(e))
$$

is an isomorphism follows from the last proposition and the following wellknown result: Given a ring $T$ with nilpotent radical nil $T$, the natural map $G_{0}(T / \operatorname{nil} T) \rightarrow G_{0}(T)$ is an isomorphism.

We call the diagram above the fundamental diagram. Applying the exact functor ()$^{G}$ to the exact sequence $0 \rightarrow \underline{b} \rightarrow S \rightarrow S / \underline{b} \rightarrow 0$ it follows that $(S / \underline{b})^{G} \cong R / \underline{b} \cap R$.

We remark that $\underline{m}_{R}$ is the only ramified prime if and only if $G$ acts freely, i.e. $\sigma(x)=x$ iff $\sigma=I$ or $x=0$. Moreover this is equivalent to $R$ being an isolated singularity, i.e. $R_{\underline{p}}$ being regular for all nonmaximal prime ideals $\underline{p}$. We have the following characterization of this.

Theorem 2.3. $R$ is an isolated singularity if and only if $\operatorname{Ker}\left(G_{0}(S[G]) \rightarrow G_{0}(R)\right)$ $=[\mathrm{C}] G_{0}(S[G])$.

Proof. Suppose that $G$ acts freely. Then $R$ is an isolated singularity and $\sqrt{\underline{a}}=$ $\underline{m}_{S}$ where

$$
\underline{a}=\operatorname{Ker}(S \rightarrow S[G] /(e)) .
$$

Observing that $S / \underline{m}_{S} \cong \mathbf{C}$, the fundamental diagram with $\underline{b}=\underline{m}_{s}$ becomes

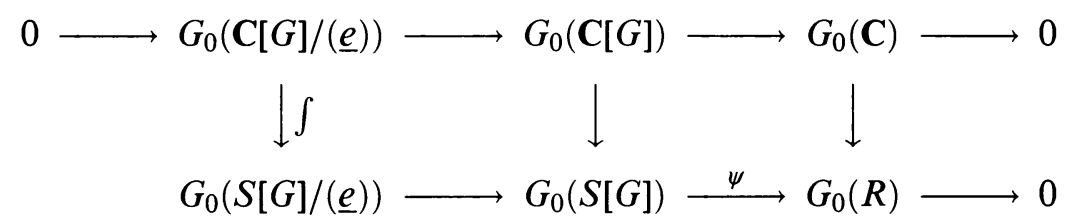

It is clear that the classes of finite $S[G]$-modules generate $\operatorname{Ker} \psi$. One sees even that the classes $[W]$ of $\mathbf{C}[G]$-modules $W$ with $W$ indecomposable, nontrivial, form a basis of $\operatorname{Ker} \psi$. that

We prove now the other implication. Auslander and Reiten proved in [5]

$$
\operatorname{rank} G_{0}(R)=\operatorname{rank} G_{0}(S)=1
$$


Suppose that $G$ does not act freely. Then we will prove that

$$
\operatorname{rank} G_{0}(S[G]) /[C] G_{0}(S[G])>1,
$$

so it cannot be isomorphic to $G_{0}(R)$.

If $G$ does not act freely then there is a prime $p \nsubseteq \underline{m}_{R}$ which is ramified. We have that the canonical epimorphism $G_{0}(S[G]) \rightarrow G_{0}\left(S_{p}[G]\right) \rightarrow$ 0 contains in its kernel the classes of all finite length modules, as $M_{\underline{p}}=0$ for all finite length modules, $\underline{p}$ a nonmaximal prime ideal. Therefore we get an epimorphism $G_{0}(S[G]) /[C] G_{0}(S[G]) \rightarrow G_{0}\left(R_{p}\right) \rightarrow 0$. Since $\left(S_{\underline{p}}\right)^{G}=$ $R_{\underline{p}}$ is ramified in $S_{\underline{p}}$, Theorem 1.3 shows that $\operatorname{rank} G_{0}\left(S_{\underline{p}}[G]\right)>1$, hence $\operatorname{rank} G_{0}(S[G]) /[C] G_{0}(S[G])>1$.

We recall some more facts proved in [4]. $G_{0}(\mathbf{C}[G])$ has a well-known ring structure with product $\left[W_{1}\right] \cdot\left[W_{2}\right]=\left[W_{1} \otimes_{\mathbf{C}} W_{2}\right]$ for $\mathbf{C}[G]$-modules $W_{1}$ and $W_{2}$. The map $\beta: G_{0}(S[G]) \rightarrow G_{0}(\mathbf{C}[G])$ given by $\beta([P])=[P / \operatorname{rad} P]$ for $P$ in $\underline{P} S[G]$, is a ring isomorphism. Furthermore $\beta([\mathbf{C}])=\sum_{i=1}^{n}(-1)^{i}\left[\Lambda^{i} V\right]$ where $\overline{\bar{V}}$ is the $n$-dimensional module induced by the inclusion of $G$ in $G L(n, \mathbf{C})=$ $G L(V)$.

From these facts and Theorem 2.3, we obtain the following Corollary:

Corollary. The following are equivalent:

(1) $G_{0}(R) \cong G_{0}(\mathbf{C}[G]) /\left(\sum(-1)^{i}\left[\Lambda^{i} V\right] G_{0}(\mathbf{C}[G])\right)$,

(2) $\operatorname{rank} G_{0}(\mathbf{C}[G]) /\left(\sum(-1)^{i}\left[\Lambda^{i} V\right] G_{0}(\mathbf{C}[G])\right)=1$,

(3) $G$ acts freely on $V$.

(4) $\operatorname{Ker}\left(G_{0}(S[G]) \rightarrow G_{0}(R)\right)=[\mathbf{C}] \cdot G_{0}(S[G])$.

If $R$ is an isolated singularity, $\operatorname{Ker} \psi$ is an ideal and hence $G_{0}(R)$ has a ring structure. We give now a condition on the $R$-module $S$ that is equivalent to $\operatorname{Ker} \psi$ being an ideal.

Definition. Let $T$ be an integrally closed commutative domain and $L$ a reflexive $T$-module.

(1) We call $L$ a Grothendieck module if the map Obj $\operatorname{Ref} T \rightarrow G_{0}(T)$ given by $M \rightarrow[M \cdot L]=\left[\left(M \otimes_{R} L\right)^{* *}\right]$ is additive, in the sense that for every exact sequence, $0 \rightarrow M_{1} \rightarrow M_{2} \rightarrow M_{3} \rightarrow 0$, of reflexive $T$-modules, one has $\left[M_{2} \cdot L\right]=\left[M_{1} \cdot L\right]+\left[M_{3} \cdot L\right]$.

(2) We denote by $A(T)$ the subgroup of $G_{0}(T)$ generated by the classes of Grothendieck modules.

(3) If $A(T)=G_{0}(T)$ we call $T$ a Grothendieck ring and in this case each family $\left(L_{i}\right)_{i \in I}$ of Grothendieck modules generating $G_{0}(T)$ is called a Grothendieck family.

Proposition 3.1. Let $T$ be an integrally closed domain.

(1) $A(T)$ has a ring structure, with identity [T] and product $\left[L_{1}\right]\left[L_{2}\right]=$ $\left[L_{1} \cdot L_{2}\right]$ for $L_{1}$ and $L_{2}$ Grothendieck modules.

(2) $G_{0}(T)$ is an $A(T)$-module if we define $[L] \cdot[M]=[L \cdot M]$ for all Grothendieck modules $L$ and all reflexive modules $M$.

Proof. Fix a Grothendieck module $L$. Since the map Obj Ref $-\bmod T \rightarrow$ $G_{0}(T)$ is additive, we have a group endomorphism $G_{0}(T) \stackrel{[L]^{\circ}}{\rightarrow} G_{0}(T)$ given by 
$[M] \rightarrow[L \cdot M]$ for any reflexive module $M$. We claim that if $L_{1}$ and $L_{2}$ are Grothendieck modules then $L_{1} \cdot L_{2}$ is a Grothendieck module. Namely, if $0 \rightarrow A \rightarrow B \rightarrow C \rightarrow 0$ is an exact sequence of reflexive $T$-modules, then $\left.\left[\left(L_{1} \cdot L_{2}\right) \cdot B\right]=\left[L_{1} \cdot\left(L_{2} \cdot B\right)\right]=\left[L_{1}\right] \cdot\left[L_{2} \cdot B\right]=\left[L_{1}\right]\left(\left[L_{2} \cdot A\right]\right)+\left[L_{2} \cdot C\right]\right)=$ $\left[L_{1}\right] \cdot\left[L_{2} \cdot A\right]+\left[L_{1}\right] \cdot\left[L_{2} \cdot C\right]=\left[\left(L_{1} \cdot L_{2}\right) \cdot A\right]+\left[\left(L_{1} \cdot L_{2}\right) \cdot C\right]$, which proves the claim.

It follows easily that the map $A(T) \times A(T) \rightarrow A(T)$ given by

$$
\left(\sum \lambda_{i}\left[L_{i}\right]\right)\left(\sum \mu_{j}\left[L_{j}\right]\right)=\sum \lambda_{i} \mu_{j}\left[L_{i} \cdot L_{j}\right]
$$

for $\lambda_{i}$ and $\mu_{j} \in \mathbf{Z}, L_{i}$ and $L_{j}$ Grothendieck modules is well defined and makes $A(T)$ a ring with unit $[T]$. Moreover it follows that $G_{0}(T)$ is an $A(T)$ module.

Theorem 3.2. Let $R=S^{G}$ be a quotient singularity. Then

$$
\operatorname{Ker}\left(G_{0}(S[G]) \rightarrow G_{0}(R)\right)
$$

is an ideal iff the summands of the R-module $S$ are Grothendieck modules.

Proof. Suppose that $\operatorname{Ker} \psi$ is an ideal. We use the epimorphism $\psi: G_{0}(S[G])$ $\rightarrow G_{0}(R)$, to put a ring structure on $G_{0}(R)$, whose multiplication we denote by " $\times$ ". Now fix a summand $L$ of $S$ and a reflexive $R$-module $M$. Then $L=P^{G}$ and $M=N^{G}$ where $P \in \underline{P} S[G]$ and $N \in \operatorname{Ref} S[G]$, see [4]. Thus $\psi\left(P \otimes_{S} N\right)=[L \cdot M]=\psi([P]) \times \psi([\bar{M}])=[L] \times[M]$. Hence, if $0 \rightarrow A \rightarrow B \rightarrow$ $C \rightarrow 0$ is an exact sequence of reflexive $R$-modules, then $[L \cdot B]=[L] \times[B]=$ $[L] \times([A]+[C])=[L \cdot A]+[L \cdot C]$ i.e. $L$ is a Grothendieck module.

Now suppose that every summand of $S$ is a Grothendieck $R$-module. Then the last theorem says that $G_{0}(R)$ has a ring structure where $\left[L_{1}\right] \cdot\left[L_{2}\right]=\left[L_{1} \cdot L_{2}\right]$ for $L_{1}$ and $L_{2}$ summands of $S$.

Let $L_{1}=P_{1}^{G}, L_{2}=P_{2}^{G}$ where $P_{1}$ and $P_{2}$ are in $\underline{\underline{P}} S[G]$. As we have $\psi\left(\left[P_{1} \otimes_{S} P_{2}\right]\right)=\left[L_{1} \cdot L_{2}\right], \psi$ is a ring map and consequently $\operatorname{Ker} \psi$ is an ideal.

As we have seen in the beginning $[C] G_{0}(S[G]) \subset \operatorname{Ker} \psi \subset \underline{J}$. The equality $\operatorname{Ker} \psi=\underline{J}$ holds exactly when the canonical epimorphism

$$
G_{0}(R) \rightarrow \mathbf{Z} \amalg \mathrm{Cl}(R) \rightarrow 0
$$

is an isomorphism.

Our next result has the following consequence. If for an integrally closed domain $T$ the map $G_{0}(T) \rightarrow \mathrm{Z} \amalg \mathrm{Cl}(T) \rightarrow 0$ is an isomorphism, then $T$ is a Grothendieck ring and every reflexive $T$-module is a Grothendieck module.

Proposition 3.3. Let $T$ be an integrally closed domain and $\left\{\underline{p}_{\alpha}\right\}_{\alpha \in I}$ a family of prime ideals in $T$ such that $\left[T / \underline{p}_{\alpha}\right]=0$ in $G_{0}(T)$ for all $\alpha \in I$. If $M$ is a reflexive $T$-module such that $M_{\underline{q}}$ is $T_{q}$-free for every prime ideal $q$ with $\underline{q} \notin\left\{p_{\alpha}\right\}_{\alpha \in I}$, then $M$ is a Grothendieck module. Moreover, in this case $[M \cdot N]=$ $[M] \cdot[N]=\left[M \otimes_{R} N\right]$ for all reflexive $T$-modules $N$.

Proof. From an exact sequence $0 \rightarrow A \rightarrow B \rightarrow C \rightarrow 0$ of reflexive $T$-modules, we obtain an exact sequence $A \cdot M \rightarrow B \cdot M \rightarrow C \cdot M$, and

$$
\operatorname{Supp}(\operatorname{Ker}(A \cdot M \rightarrow B \cdot M))
$$


as well as

$$
\operatorname{Supp}(\operatorname{Coker}(B \cdot M \rightarrow C \cdot M))
$$

are contained in $\left\{\underline{p}_{\alpha}: \alpha \in I\right\}$. It is known that given a $T$-module $L$ there is a composition series $\left(L_{i}\right)_{0 \leq i \leq n}$ s.t. for $0 \leq i \leq n-1, L_{i} / L_{i+1} \cong T / \underline{q}_{i}$ where each $\underline{q}_{i}$ is a prime ideal in supp $L$. (See for instance IV, 4.2 of [8].) Hence it follows that the class $[L]$ of a module $L$ is contained in the subgroup of $G_{0}(T)$ generated by $\left\{\left[T / \underline{q}_{i}\right]: \underline{q}_{i} \in \operatorname{supp} L\right\}$. As $\left[T / \underline{p}_{i}\right]=0$ in our case, we get $[A \cdot M]+[C \cdot M]=[B \cdot M]$, i.e. $M$ is a Grothendieck module.

Since the natural map $(M \otimes N)_{q} \rightarrow\left(M_{q} \cdot N_{q}\right)$ is an isomorphism for $\underline{q} \notin$ $\left\{\underline{p}_{\alpha}\right\}_{\alpha \in I}$, the same argument shows that $[\bar{M} \otimes \bar{N}]=[M \cdot N]$.

Corollary 3.4. Let $R=S^{G}$ be a quotient singularity. Then the subgroup of $G_{0}(R)$ generated by the classes $[M]$ of modules $M$ which are free outside the maximal ideal is a subring of $A(R)$. Its identity is $[R]$ and the product is defined by $[M] \cdot[N]=[M \otimes N]=[M \cdot N]$ for all modules $M$ and $N$ which are free outside the maximal ideal.

Proof. This is a consequence of the proposition, as $\left[R / m_{R}\right]=[\mathbf{C}]=0$ in $G_{0}(R)$.

Corollary 3.5. Suppose that $T$ is an integrally closed domain and that the canonical epimorphism between $G_{0}(T)$ and $\mathbf{Z} \cdot[T] \amalg \mathrm{Cl}(T)$ is an isomorphism. Then every reflexive module is a Grothendieck module. Moreover the family of rank one reflexive modules is a Grothendieck family.

Proof. To say that the canonical epimorphism is an isomorphism is equivalent to $[T / \underline{p}]=0$ for all prime ideals $\underline{p}$ with $\mathrm{ht} \underline{p} \geq 2$, see [8]. Since for every reflexive $T$-module $M$ it holds that $M_{p}$ is free for ht $p \leq 1$, it follows, from Proposition 3.3, that every reflexive module is Grothendieck. Moreover, since in this case the classes of reflexive rank one modules generate $G_{0}(T)$, they form a Grothendieck family.

Remark. (1) If $A$ and $B$ are rank one reflexive modules and if $C(M)$ denotes the divisor associated with $M$, one has $C(A \otimes B)=C(A)+C(B)$, see [8]. It follows that if $G_{0}(T) \cong \mathrm{Z} \amalg \mathrm{Cl}(T)$ then the product is given by $(m, \alpha)\left(m^{\prime}, \alpha^{\prime}\right)$ $=\left(m m^{\prime}, m \alpha^{\prime}+m^{\prime} \alpha\right)$ for $m$ and $m^{\prime}$ in $\mathbf{Z}, \alpha$ and $\alpha^{\prime}$ in $\operatorname{Cl}(T)$.

Corollary 3.6. A Dedekind domain is a Grothendieck ring.

\section{ChAPTER II. GROUPS ACTING FREELY ON SUBSPACES, ONE RAMIFIED LINE AND FORMAL POWER SERIES}

In this chapter we use the fundamental diagram to get an exact sequence of the form

$$
0 \rightarrow G_{0}\left(\mathbf{C}\left[\left[V_{2}\right]\right]^{G}\right) / \mathbf{Z}\left[\mathbf{C}\left[\left[V_{2}\right]\right]\right] \rightarrow G_{0}(R) \rightarrow G_{0}\left(\mathbf{C}\left[\left[V_{1}\right]\right]^{G}\right) \rightarrow 0
$$

in the case where $G$ acts freely on $V_{1}$ and $V$ has a decomposition, as $\mathrm{C}[G]$ module, of the form $V=V_{1} \amalg_{\mathrm{C}[G]} V_{2}$.

We can use this sequence to get information about $G_{0}(R)$. For instance we show that, in this case, $G_{0}(R) \cong \mathrm{Z}$ iff the following three conditions are satisfied: 
(1) $\operatorname{dim} V_{1}=2$.

(2) $G$ is the binary icosahedral group.

(3) $G$ acts trivially on $V_{2}$.

If the ramification locus consists of one line, we prove that $\operatorname{Ker} \psi$ is an ideal if and only if $G$ acts trivially on this line.

In the last section we prove that if $R$ is a quotient singularity, then the natural map

$$
\theta: G_{0}(R) \rightarrow G_{0}(R[[t]]),
$$

given by $[M] \rightarrow\left[M \otimes_{R} R[[t]]\right]$ for $M$ an $R$-module, is an isomorphism. This is not true for a general ring. A counterexample is provided by the ring $T=$ $Q(u)[[x, y, z]] /\left(x^{2}+y^{3}+u z^{6}\right)$.

We suppose in this section that the group $G$ acts on

$$
V=\mathbf{C} x_{1} \amalg_{\mathbf{C}} \cdots \amalg_{\mathbf{C}} \mathbf{C} x_{n}, \quad n \geq 2,
$$

without pseudo-reflections and that $V$ admits a $\mathrm{C}[G]$-module decomposition $V=V_{1} \amalg_{\mathbf{C}[G]} V_{2}$, where $V_{1}=\mathbf{C} x_{1}, \amalg_{\mathbf{C}} \cdots \amalg_{\mathbf{C}} \mathbf{C} x_{k}, V_{2}=\mathbf{C} x_{k+1} \amalg_{\mathbf{C}} \cdots \amalg_{\mathbf{C}} \mathbf{C} x_{n}$ with $k<n, G$ acting freely on $V_{1}$.

Theorem 1.1. There is an exact sequence

$$
\begin{aligned}
0 & \rightarrow G_{0}\left(R /\left(x_{1}, \ldots, x_{k}\right) R\right) / \mathbf{Z}\left[S /\left(x_{1}, \ldots, x_{k}\right)\right] \\
& \stackrel{t}{\rightarrow} G_{0}(R) \stackrel{\alpha}{\rightarrow} G_{0}\left(R /\left(x_{k+1}, \ldots, x_{n}\right) \cap R\right) \rightarrow 0
\end{aligned}
$$

where $t$ is induced by the canonical epimorphism of rings $R \rightarrow R /\left(x_{1}, \ldots, x_{k}\right) \cap$ $R$ and $\alpha$ is given by

$$
\alpha\left(\left[\left(S \otimes_{\mathbf{C}} W\right)^{G}\right]\right)=\left[\left(S /\left(x_{k+1}, \ldots, x_{n}\right) \otimes_{\mathbf{C}} W\right)^{G}\right]
$$

for any $\mathbf{C}[G]$-module $W$.

Proof. The proof uses the isomorphism $G_{0}(\mathbf{C}[G]) \cong G_{0}(S[G])$ and the following lemma.

Lemma 1.2. Suppose that $G \neq\{1\}$ acts freely on a $\mathbf{C}$-vector space $V$. Take in $G_{0}(\mathbf{C}[G])$ the element $a=\sum_{i=0}^{\operatorname{dim} V}(-1)^{i}\left[\Lambda^{i} V\right]$ and set $(\underline{a})=a \cdot G_{0}(\mathbf{C}[G])$. Then $\operatorname{ann}(\underline{a})=[\mathbf{C}[G]] G_{0}(\mathbf{C}[G])=\mathbf{Z} \cdot[\mathbf{C}[G]]$.

Proof. The equality $[\mathbf{C}[G]] \cdot G_{0}(\mathbf{C}[G])=\mathbf{Z} \cdot[\mathbf{C}[G]]$ comes from the fact that $W \otimes_{\mathbf{C}} \mathbf{C}[G] \cong(\operatorname{dim} W) \mathbf{C}[G]$ for any $\mathbf{C}[G]$-module $W$. We now show the other equality.

First assume in addition that $G$ is cyclic. Then $G_{0}(\mathbf{C}[G] \cong Z[\langle\sigma\rangle]$ where $\sigma$ is a generator of $G^{*}=\operatorname{Hom}(G, C-\{0\})$. Using this isomorphism as an identification, one has $\underline{a}=(\sigma-1)^{n}=\Delta^{n}$, the $n$th power of the augmentation ideal and $[\mathbf{C}[G]]=\sum_{i=0}^{|G|-1} \sigma^{i}$.

We use induction on $n$.

If $n=1$, then the result is clear.

Suppose that the result is known for $n-1, n>1$.

It is clear that $\left(\sum_{i=0}^{|G|-1} \sigma^{i}\right)(\sigma-1)^{n}=0$, so $\mathbf{Z} \cdot\left(\sum_{i=0}^{|G|-1} \sigma^{i}\right) \subset$ ann $(\sigma-1)^{n}$. Suppose that $f(\sigma-1)^{n}=0$. Then $f(\sigma-1)^{n-1}(\sigma-1)=0$ and therefore 
$f(\sigma-1)^{n-1} \in(\sigma-1) \cap \operatorname{ann}(\sigma-1)=\{0\}$. Hence $f(\sigma-1)^{n-1}=0$ and by the induction hypothesis

$$
f \in\left(\sum_{i=0}^{|G|-1} \sigma^{i}\right) \mathbf{Z}[\langle\sigma\rangle]=\mathbf{Z} \cdot\left(\sum_{i=1}^{|G|-1} \sigma\right) .
$$

Now suppose that $G$ is an arbitrary finite group.

Let $\operatorname{dim}: G_{0}(\mathbf{C}[G]) \rightarrow \mathbf{Z}$ be the map given by $\operatorname{dim}[W]=\operatorname{dim}_{\mathbf{C}} W$ for $W$ a $\mathbf{C}[G]$-module. If $f \in G_{0}(\mathbf{C}[G])$ then $f \cdot(\mathbf{C}[G])=(\operatorname{dim} f)[\mathbf{C}[G]]$. So $f$. $[\mathbf{C}[G]]=0$ if and only if $\operatorname{dim} f=0$ which shows that $[\mathbf{C}[G]] G_{0}(\mathbf{C}[G]) \subset$ $\operatorname{ann}(\underline{a})$. Now suppose $b \cdot a=0, b \in G_{0}(\mathbf{C}[G])$. Denote by $\operatorname{res}_{\langle\sigma\rangle}^{G}$ the natural map $G_{0}(\mathbf{C}[G]) \rightarrow G_{0}(\mathbf{C}[\langle\sigma\rangle])$ for $\sigma \in G$. Then for every $\sigma \in G$ we have, by the first part, $\operatorname{res}_{\langle\sigma\rangle}^{G} b=m\langle\sigma\rangle[\mathbf{C}[\langle\sigma\rangle]]$ with $m \in \mathbf{Z}$. Hence

$$
\operatorname{res}_{\langle\sigma\rangle}^{G} b=\frac{\operatorname{dim} b}{|\sigma|} \cdot[\mathbf{C}[\langle\sigma\rangle]] .
$$

Take in $G_{0}(\mathbf{C}[G])$ the element $\tilde{b}=(\operatorname{dim} b)[\mathbf{C}[G]]$. Then for every $\sigma \in G$ we have

$$
\operatorname{res}_{\sigma}^{G} \tilde{b}=(\operatorname{dim} b) \frac{|G|}{|\langle\sigma\rangle|}[\mathbf{C}[\langle\sigma\rangle]]=|G| \operatorname{res}_{\langle\sigma\rangle}^{G} b .
$$

Now since we have a ring monomorphism

$$
0 \rightarrow G_{0}(\mathbf{C}[G]) \stackrel{\text { ע res}}{\rightarrow} \coprod_{\sigma \in G} G_{0}(C\langle\sigma\rangle)
$$

it follows that $\tilde{b}=|G| \cdot b$ therefore $|G| b=(\operatorname{dim} b)[\mathbf{C}[G]]$. Taking the basis $\left\{\left[W_{2}\right], \ldots,\left[W_{k}\right],[\mathbf{C}[G]]\right\}$ of $G_{0}(\mathbf{C}[G])$, where $W_{i}$ are all the irreducible nontrivial representations of $G$, we have $b=\lambda_{2}\left[W_{2}\right]+\cdots+\lambda_{k}\left[W_{k}\right]+u[\mathbf{C}[G]]$ for some $\lambda_{i}$ 's and $u$ in $\mathbf{Z}$.

Therefore

$$
|G| \cdot b=(\operatorname{dim} b)[\mathbf{C}[G]]=|G| \cdot\left(\lambda_{2}\left[W_{2}\right]+\cdots+\lambda_{k}\left[W_{k}\right]+u[\mathbf{C}[G]]\right) .
$$

It follows that $\lambda_{i}=0$ for $i=2, \ldots, k$, so $b=u[\mathbf{C}[G]] \in \mathbf{Z}[\mathbf{C}[G]]$.

Proof of the Theorem. Applying the fundamental diagram with the ideal $\left(x_{1}, \ldots, x_{k}\right)$, we obtain

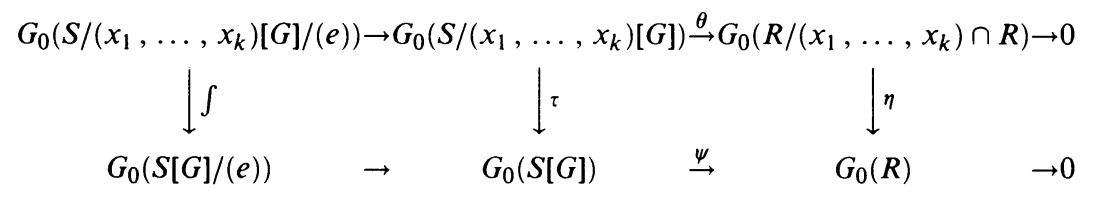

and it follows from diagram chasing that $\tau / \operatorname{Ker} \theta: \operatorname{Ker} \theta \rightarrow \operatorname{Ker} \psi$ is an epimorphism. But $\operatorname{rank} G_{0}(R)=\operatorname{rank} G_{0}\left(R /\left(x_{1}, \ldots, x_{k}\right) \cap R\right)=1$ and

$$
G_{0}\left(S /\left(x_{1}, \ldots, x_{k}\right)[G]\right) \cong G_{0}(\mathbf{C}[G]) \cong G_{0}(S[G]) .
$$

Hence $\operatorname{Ker} \theta$ and $\operatorname{Ker} \tau$ are free abelian groups of the same rank and it follows that $\left.\tau\right|_{\operatorname{Ker} \theta}: \operatorname{Ker} \theta \rightarrow \operatorname{Ker} \psi$ is an isomorphism. 
Then we have the following commutative diagram:

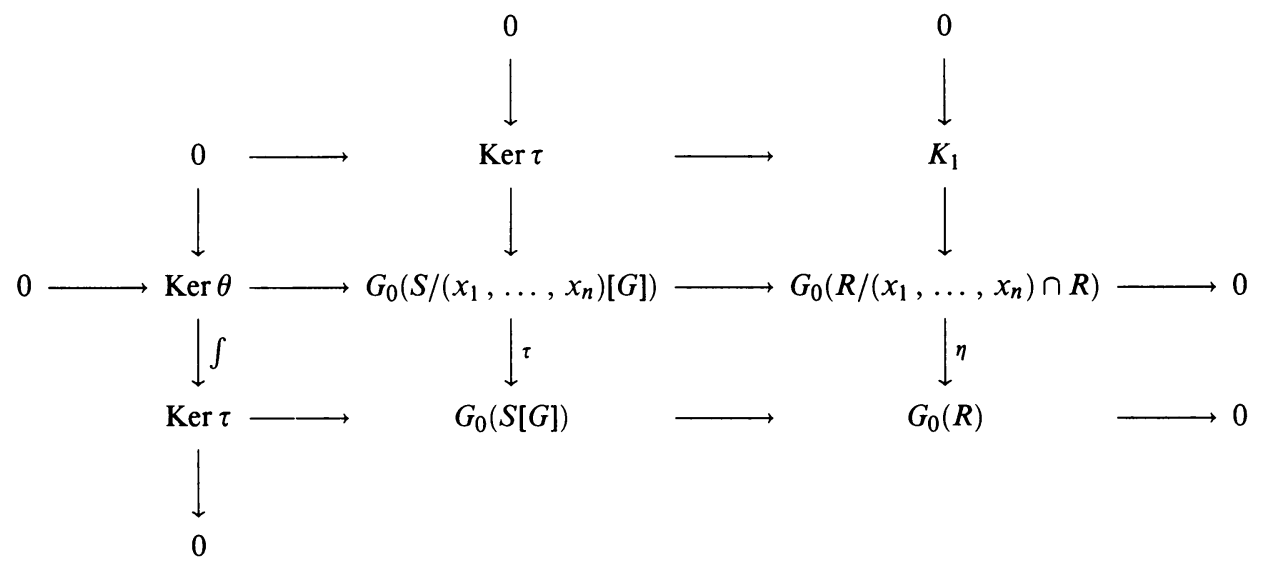

From the snake lemma it follows that $\operatorname{Ker} \tau \rightarrow K_{1}$ is an epimorphism. Also, diagram chasing shows that $\operatorname{coker} \tau \rightarrow \operatorname{coker} \eta$ is an isomorphism. We also have a commutative diagram:

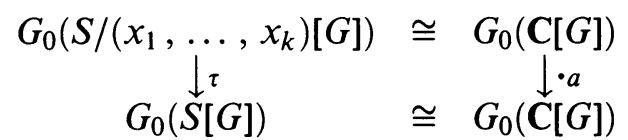

where $a=\sum_{i=0}^{k}(-1)^{i}\left[\Lambda^{i} V_{1}\right]$. We know by Lemma 1.2 that $\operatorname{ann}(\underline{a})=\mathbf{Z}$. $[\mathbf{C}[G]]$. Then $\operatorname{Ker} \tau=\mathbf{Z} \cdot\left[S /\left(x_{1}, \ldots, x_{k}\right)[G]\right]$. Since $\left(S /\left(x_{1}, \ldots, x_{k}\right)[G]\right)^{G} \cong$ $S /\left(x_{1}, \ldots, x_{k}\right)$ it follows that

$$
K_{1}=\mathbf{Z} \cdot\left[S /\left(x_{1}, \ldots, x_{k}\right)\right] .
$$

So we get an exact sequence

$$
\begin{aligned}
0 & \rightarrow \mathbf{Z} \cdot\left[S /\left(x_{1}, \ldots, x_{k}\right)\right] \rightarrow G_{0}\left(R /\left(x_{1}, \ldots, x_{k}\right) \cap R\right) \\
& \stackrel{t}{\rightarrow} G_{0}(R) \stackrel{p}{\rightarrow} G_{0}(\mathbf{C}[G]) /(\underline{a}) \rightarrow 0
\end{aligned}
$$

where $p$ is given by $p\left[\left(S \otimes_{\mathbf{C}} W\right)^{G}\right)=[W]+(\underline{a})$. Therefore $p$ corresponds to the map of the statement via the isomorphism

$$
G_{0}\left(R /\left(x_{k+1}, \ldots, x_{n}\right) \cap R\right) \cong G_{0}(\mathbf{C}[G]) /(\underline{a}) .
$$

Remark. Since

$$
\alpha\left(\left[(S \otimes W)^{G}\right]\right)=\left[\left(S /\left(x_{k+1}, \ldots, x_{n}\right) \otimes W\right)^{G}\right],
$$

we obtain a commutative diagram

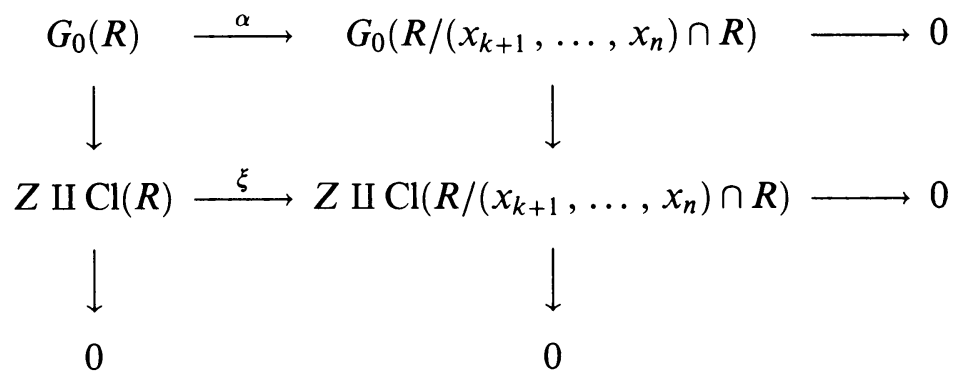


Moreover, if $k>1$, the map $\xi$ is an isomorphism, as in this case

$$
\mathrm{Cl}(R) \cong \mathrm{Cl}\left(R /\left(x_{k+1}, \ldots, x_{n}\right) \cap R\right) \cong \operatorname{Hom}(G, \mathbf{C}-\{0\}), \quad \text { see [4] }
$$

Corollary 3.1. Suppose that $V=V_{1} \amalg_{\mathbf{C}[G]} V_{2} ; V_{1}=\mathbf{C} x_{1} \amalg_{\mathbf{C}} \cdots \amalg_{\mathbf{C}} \mathbf{C} x_{k} ; V_{2}=$ $\mathbf{C} x_{k+1} \amalg_{\mathbf{C}} \cdots \amalg_{\mathbf{C}} \mathbf{C} x_{n}$ and that

(1) $G$ acts freely on $V_{1}$.

(2) $\mathbf{C}\left[\left[V_{2}\right]\right]^{G}$ is regular. (This holds iff $\operatorname{dim} V_{2}=1$ or $\operatorname{Im}\left(G \rightarrow G L\left(V_{2}\right)\right)$ is generated by pseudo-reflections.)

Then we have an exact sequence

$$
0 \rightarrow \operatorname{Ker} \alpha \rightarrow G_{0}(R) \rightarrow G_{0}\left(R /\left(x_{k+1}, \ldots, x_{k}\right) \cap R\right) \rightarrow 0
$$

where $\operatorname{Ker} \alpha$, generated by $\left[R /\left(x_{1}, \ldots, x_{k}\right) \cap R\right]$ is cyclic of order $\mid \operatorname{Im}(G \rightarrow$ $\left.G L\left(V_{2}\right)\right)$.

Moreover, if we assume in addition, that $G$ acts trivially on $V_{2}$ then $G_{0}(R) \cong$ $G_{0}\left(\mathbf{C}\left[\left[V_{1}\right]\right]^{G}\right)$.

Proof. In this case

$$
G_{0}\left(R /\left(x_{1}, \ldots, x_{n}\right) \cap R\right)=\mathbf{Z} \cdot\left[R /\left(x_{1}, \ldots, x_{k}\right) \cap R\right]
$$

and

$$
\operatorname{Rank}_{R /\left(x_{1}, \ldots, x_{k}\right) \cap R}\left(S /\left(x_{1}, \ldots, x_{k} \cap R\right)\right)=\left|\operatorname{Im}\left(G \rightarrow G L\left(V_{2}\right)\right)\right| .
$$

The assertion follows using the exact sequence of the theorem.

Moreover, if $G$ acts trivially on $V_{2}$ then $\operatorname{Im}\left(G \rightarrow G L\left(V_{2}\right)\right)=\{I\}$.

\section{Applications}

We now give some applications of the sequence of Theorem 1.1.

For the next proposition we need the following results, found in the book of J. A. Wolf [17].

Theorem 2.1 [17]. (a) Let $G$ be a finite group which admits a free C-representation. Let $\psi_{G}(\mathbf{C})$ denote the set of all equivalence classes of irreducible free representations of $G$. Then the elements of $\psi_{G}(\mathbf{C})$ have the same degree $\delta(G)$.

(b) If, moreover, $G=[G, G]$ (the commutator subgroup of $G$ ) then $\delta(G)=2$ and $G$ is the binary icosahedral group, $I^{*}$.

Proposition 2.2. Suppose that $G$ admits a free representation and suppose that $\delta(G)=2$ or 1 . Suppose $G$ acts freely on $V$ where $\operatorname{dim} V=2 n$ and $R=$ $\mathrm{C}[[V]]^{G}$. Then $\mid$ Torsion $\left.G_{0}(R)|=| G\right|^{n-1} \cdot\left|G^{*}\right|^{n}$.

Proof. We use induction on $n$.

If $n=1$, we know that $G_{0}(R) \cong \mathrm{Z} \amalg G^{*} \cong \mathrm{Z} \amalg \mathrm{Cl}(R)$.

Suppose the result is valid for $n-1, n>1$. Then using Theorem 2.1 we decompose $V=V_{1} \amalg_{\mathrm{C}[G]} V_{2}$ where $\operatorname{dim} V_{1}=2$. So we have by Theorem 1.1 an exact sequence

$$
0 \rightarrow G_{0}\left(\mathbf{C}\left[\left[V_{1}\right]\right]^{G}\right) / \mathbf{Z}\left[\mathbf{C}\left[\left[V_{1}\right]\right]\right] \rightarrow \operatorname{Tors}\left(G_{0}(R)\right) \rightarrow \operatorname{Tors} G_{0}\left(\mathbf{C}\left[\left[V_{2}\right]\right]^{G}\right) \rightarrow 0
$$

where Tors means torsion. By the induction hypothesis

$$
\mid \text { Tors }\left.G_{0}\left(\mathbf{C}\left[\left[V_{2}\right]\right]^{G}\right)|=| G\right|^{n-2} \cdot\left|G^{*}\right|^{n-1},
$$


and we have to prove that

$$
\left|G_{0}\left(\mathbf{C}\left[\left[V_{1}\right]\right]^{G}\right) / \mathbf{Z}\left[\mathbf{C}\left[\left[V_{1}\right]\right]\right]\right|=|G|\left|G^{*}\right| .
$$

We have $G_{0}\left(\mathbf{C}\left[\left[V_{1}\right]\right]^{G}\right) \cong \mathbf{Z} \amalg G^{*}$ and, using this isomorphism as identification, $\left[\mathrm{C}\left[\left[V_{1}\right]\right]\right]=(|G|, \beta)$ for some $\beta$.

Hence we have an exact sequence,

$$
\begin{aligned}
0 \rightarrow \mathbf{Z} & \rightarrow \mathbf{Z} \amalg G^{*} \rightarrow G_{0}\left(\mathbf{C}\left[\left[V_{1}\right]\right]^{G}\right) / \mathbf{Z} \cdot\left[\mathbf{C}\left[\left[V_{1}\right]\right]\right] \rightarrow 0 \\
1 & \rightarrow(|G|, \beta) .
\end{aligned}
$$

Therefore we get an exact sequence

$$
\begin{aligned}
0 & \rightarrow \operatorname{Hom}\left(\mathbf{Z} \amalg G^{*}, \mathbf{Z}\right) \stackrel{\cdot|G|}{\rightarrow} \operatorname{Hom}(\mathbf{Z}, \mathbf{Z}) \\
& \rightarrow \operatorname{Ext}^{1}\left(G_{0}\left(\mathbf{C}\left[\left[V_{1}\right]\right]^{G}\right) / \mathbf{Z}\left[\mathbf{C}\left[\left[V_{1}\right]\right]\right], \mathbf{Z}\right) \rightarrow \operatorname{Ext}^{1}\left(G^{*}, \mathbf{Z}\right) \rightarrow 0
\end{aligned}
$$

so we get

$$
0 \rightarrow \mathbf{Z} /|G| \mathbf{Z} \rightarrow G_{0}\left(\mathbf{C}\left[\left[V_{1}\right]\right]^{G}\right) / \mathbf{Z}\left[\mathbf{C}\left[\left[V_{1}\right]\right]\right] \rightarrow G^{*} \rightarrow 0
$$

and we are done.

Proposition 2.3. Suppose that $V$ admits a $\mathrm{C}[G]$-module decomposition of the form $V=V_{1} \amalg_{\mathbf{C}[G]} V_{2}$ and that $G$ acts freely on $V_{1}, V_{1} \neq 0$. Then $G_{0}(R) \cong \mathbf{Z}$ if and only if the following three conditions are satisfied:

(1) $G=I^{*}$, the binary icosahedral group.

(2) $\operatorname{dim} V_{1}=2$.

(3) $G$ acts trivially on $V_{2}$.

Proof. By Corollary 1.3 we only need to prove that the conditions are necessary.

Assume that $G_{0}(R) \cong \mathbf{Z}$. Then $0=\mathrm{Cl}(R) \cong G^{*} \cong G /[G, G]$. Since $V_{1} \neq 0$ we have by Theorem 2.1 (b) that $G=I^{*}$, and from Theorem 1.1 we get an exact sequence

$$
0 \rightarrow G_{0}\left(\mathbf{C}\left[\left[V_{2}\right]\right]^{G}\right) / \mathbf{Z} \cdot\left[\mathbf{C}\left[\left[V_{2}\right]\right]\right] \rightarrow G_{0}(R) \rightarrow G_{0}\left(\mathbf{C}\left[\left[V_{1}\right]\right]^{G}\right) \rightarrow 0 .
$$

So, if $G_{0}(R) \cong \mathbf{Z}$, we get that $G_{0}\left(\mathbf{C}\left[\left[V_{2}\right]\right]\right) \cong \mathbf{Z} \cdot\left[\mathbf{C}\left[\left[V_{2}\right]\right]\right]$, which implies that $\operatorname{rank}_{\mathbf{C}\left[\left[V_{2}\right]\right] G} \mathbf{C}\left[\left[V_{2}\right]\right]=1$. Hence $G$ acts trivially on $V_{2}$. Now by Theorem 2.1, we have $\operatorname{dim} V_{1}=2 n$ and $V_{1}=V_{11} \amalg_{C[G]} \cdots \amalg_{\mathbf{C}[G]} V_{1 n}$ with $\operatorname{dim} V_{l i}=2$.

By a completely analogous argument as before we get $n=1$, i.e. $\operatorname{dim} V_{1}=2$.

Remarks. (a) Letting $I$ act freely on $V$ with $\operatorname{dim} V=2$ we get examples of quotient singularities that are UFD, but whose Grothendieck groups are not $\mathbf{Z}$.

(b) If $\delta=2$ or 1 and $G$ acts freely on $V$ we get, in an analogous way to Proposition 2.3, that $G_{0}\left(\mathbf{C}[[V]]^{G}\right) \cong \mathrm{Z} \amalg \mathrm{Cl}(R) \Leftrightarrow \operatorname{dim} R=\operatorname{dim} V=2$.

(c) It would be nice to have a characterization of those cases for which $G_{0}(R) \cong \mathbf{Z}$, (without the hypothesis of Proposition 2.3).

We finish this section with the following conjecture.

Conjecture. If $G$ acts freely on $V, \operatorname{dim} V \geq 2$ then $G_{0}(R) \cong \mathrm{Z} \amalg \operatorname{cl}(R) \Leftrightarrow$ $\operatorname{dim} V=2$.

\section{3}

In this section we give some examples and analyze when

$$
\operatorname{Ker}\left(\psi: G_{0}(S[G]) \rightarrow G_{0}(R)\right)
$$

is an ideal. 
We assume as in the section above that $V=V_{1} \amalg_{\mathbf{C}[G]} V_{2}$ and that $G$ acts freely on $V_{1}, V_{1} \neq 0$.

We introduce some notation.

Suppose $H \triangleleft G$ are finite groups. Then we have an exact dense functor

$$
()^{H}: \mathbf{C}[G]-\bmod \rightarrow \mathbf{C}[G / H]-\bmod .
$$

If we take $\operatorname{Ker}()^{H}$ to be the subcategory of modules $N$ s.t. $N^{H}=0$, and let $e_{H}=(1 /|H|) \sum_{\sigma \in H} \sigma$, we have that $\operatorname{Ker}()^{H}=\mathrm{C}[G] /\left(\underline{e}_{H}\right)-\bmod$. Hence we have the following split exact sequence

$$
0 \rightarrow G_{0}\left(\mathbf{C}[G] /\left(\underline{e}_{H}\right)\right) \rightarrow G_{0}(\mathbf{C}[G]) \rightarrow G_{0}(\mathbf{C}[G / H]) \rightarrow 0 .
$$

We remark, in addition, that $G_{0}\left(\mathbf{C}[G] / \underline{e}_{H}\right)$ is the free abelian group on the classes $[N]$ of indecomposable $\mathbf{C}[G]$-modules $N$ for which $N^{H}=N$.

Finally, observe that since $H$ is normal, $N^{H}$ is a $\mathrm{C}[G]$-submodule of $N$. Therefore, if $N$ is indecomposable, $N^{H}=N$ or $N^{H}=0$.

Proposition 3.1. Suppose that $\operatorname{Ker}\left(G \rightarrow G L\left(V_{2}\right)\right)=H \neq G$ and that $\left\{W_{1}, \ldots\right.$, $\left.W_{n}\right\}$ is a complete set of representatives of indecomposable $\mathbf{C}[G] /\left(\underline{e}_{H}\right)$-modules. If $\operatorname{Ker} \psi$ is an ideal, then the greatest common divisor of $\left\{\operatorname{dim}_{\mathbf{C}} W_{i}\right\}_{i=1}^{n}$ is larger than one.

The proof is based on the following lemma whose proof is left to the reader.

Lemma 3.2. Assume there is a commutative ring structure on $U=\mathrm{Z} \amalg T$ with identity $(1,0)$ and $T$ is a finite abelian group. Then $T=\sqrt{0}=J(U)$, where $J(U)$ is the Jacobson radical of $U$ and $\sqrt{0}$ is the nil radical.

Proof of the Proposition 3.1. Since $G \neq H$, it follows that $\left[\left(S /\left(x_{1}, \ldots, x_{k}\right)\right)^{G}\right] \neq$ 0 in $G_{0}(R)$, as

$$
\operatorname{Ker}\left(G_{0}\left(\left(S /\left(x_{1}, \ldots, x_{k}\right)\right)^{G}\right) \rightarrow G_{0}(R)\right) \cong \mathbf{Z} \cdot\left[S /\left(x_{1}, \ldots, x_{k}\right)\right] .
$$

Suppose $\operatorname{gcd}\left\{\operatorname{dim}_{\mathbf{C}} W_{i}\right\}=1$. Then there is a linear combination $\sum \lambda_{j}\left[W_{j}\right]$ with $\sum \lambda_{j} \operatorname{dim}_{\mathrm{C}} W_{j}=1$. Using the isomorphism $G_{0}(R) \cong Z \amalg T$, we get that $\varepsilon\left(\sum \lambda_{j} S \otimes W_{j}\right)=([R], \alpha)$ is invertible.

Hence there is $\beta \in G_{0}(S[G])$ s.t. $\left(\sum \lambda_{j}\left[S \otimes W_{j}\right]\right) \beta-1 \in \operatorname{Ker} \psi$. As $\operatorname{Ker} \psi$ is an ideal, we have

$$
\left(\sum(-1)^{i}\left[S \otimes \Lambda^{i} V_{1}\right]\right) \cdot\left(\sum \lambda_{j}\left[S \otimes W_{j}\right] \beta-1\right) \in \operatorname{Ker} \psi
$$

But

$$
\left(\sum(-1)^{i}\left[S \otimes \Lambda^{i} V_{1}\right]\right) \cdot\left[S \otimes W_{j}\right]=\left(S /\left(x_{1}, \ldots, x_{k}\right) \otimes W_{j}\right)
$$

therefore $\psi\left(\sum(-1)^{i}\left[S \otimes \Lambda^{i} V_{1}\right]\right) \cdot\left[S \otimes W_{j}\right]=\left[\left(S /\left(x_{1}, \ldots, x_{k}\right) \otimes W_{j}\right)^{G}\right]=0$.

It follows that $\sum(-1)^{i}\left[S \otimes \Lambda^{i} V_{1}\right] \in \operatorname{Ker} \psi$, a contradiction.

Corollary 3.3. If $\operatorname{Ker} \psi$ is an ideal and $H \neq G$ then $H \subset G^{\prime}=[G, G]$.

Proof. $H \subset \operatorname{Ker} \chi$ for all linear characters $\chi$ of $G$ so

$$
H \subset \bigcap_{\substack{\chi \text { linear } \\ \text { characters }}}(\operatorname{Ker} \chi)=G^{\prime} .
$$


We recall that in our situation, i.e. $V=V_{1} \amalg_{\mathrm{C}[G]} V_{2}, G$ acting freely on $V_{1}=\mathbf{C}_{x_{1}} \amalg \cdots \amalg \mathbf{C} x_{k}$, we have the following exact commutative diagram

$$
\begin{array}{cc}
G_{0}(\mathbf{C}[G]) \stackrel{\sim}{\longrightarrow} G_{0}\left(S /\left(x_{1}, \ldots, x_{k}\right)[G]\right) \\
\downarrow \cdot\left(\sum(-1)^{i}\left[\Lambda^{i} V_{1}\right]\right) & \downarrow \tau \\
G_{0}(\mathbf{C}[G]) \stackrel{\sim}{\longrightarrow} G_{0}(S[G]) .
\end{array}
$$

Furthermore, if $H=\operatorname{Ker}\left(G \rightarrow G L\left(V_{2}\right)\right)$, we have

$$
G_{0}(S[G]) \cong G_{0}(\mathbf{C}[G]) \cong G_{0}\left(\mathbf{C}[G] /\left(e_{H}\right)\right) \amalg G_{0}(\mathbf{C}[G / H]),
$$

and we get the commutative diagram

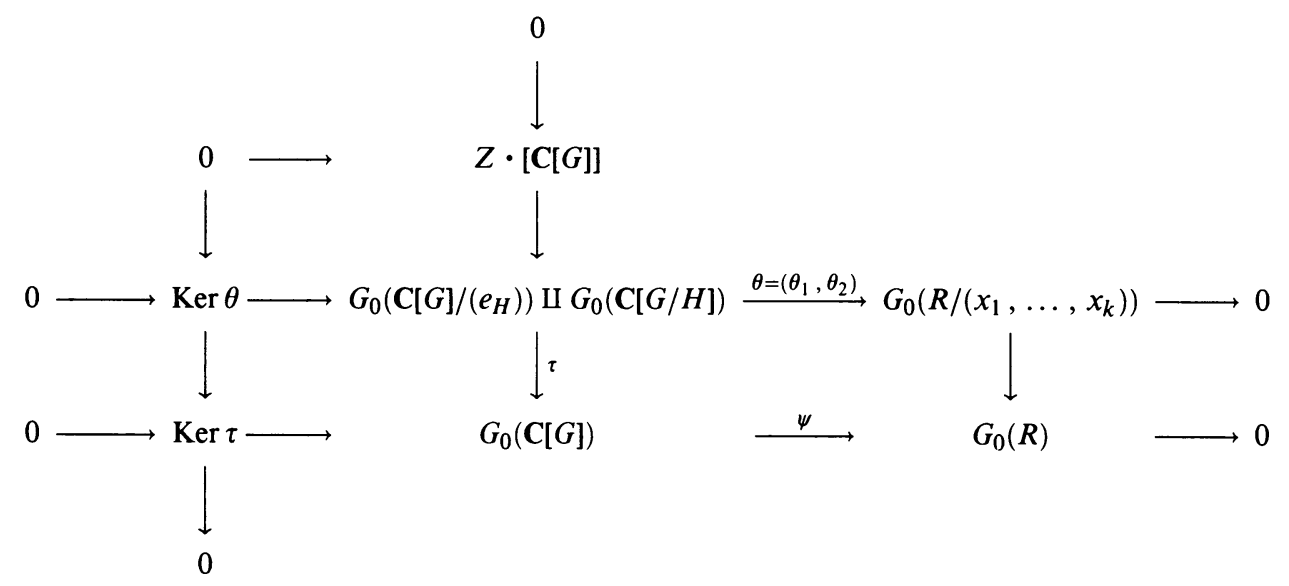

Proposition 3.4. Using the notation and remarks above, the following are equivalent:

(a) $\operatorname{Ker} \psi$ is an ideal of $G_{0}(\mathbf{C}[G])$,

(b) $Z[\mathbf{C}[G]] \amalg \operatorname{Ker} \theta$ is an ideal of $G_{0}(\mathbf{C}[G])$,

(c) $\operatorname{Ker} \theta_{2}$ is an ideal of $G_{0}(\mathbf{C}[G / H])$ and $G_{0}\left(\mathbf{C}[G] /\left(\underline{e}_{H}\right)\right) \cdot G_{0}\left(\mathbf{C}[G] /\left(\underline{e}_{H}\right)\right) \subset$ $Z[\mathbf{C}[G]] \amalg \operatorname{Ker} \theta$.

Proof. (a) $\Leftrightarrow$ (b). First we observe that $\mathbf{Z}[\mathbf{C}[G]]=\operatorname{Ker} \tau$ and $\tau(\operatorname{Ker} \theta)=$ $\operatorname{Ker} \psi$, so $\tau^{-1}(\operatorname{Ker} \psi)=\mathbf{Z}[\mathbf{C}[G]]+\operatorname{Ker} \theta=\mathbf{Z}[\mathbf{C}[G]] \amalg \operatorname{Ker} \theta$.

Since $\tau$ is a $G_{0}(\mathbf{C}[G])$-module map it follows that $\operatorname{Ker} \psi$ is an ideal if and only if $\tau^{-1}(\operatorname{Ker} \psi)=\mathbf{Z}[\mathbf{C}[G]] \amalg \operatorname{Ker} \theta$ is an ideal.

(a) and (b) imply (c). First some remarks.

We have a commutative diagram

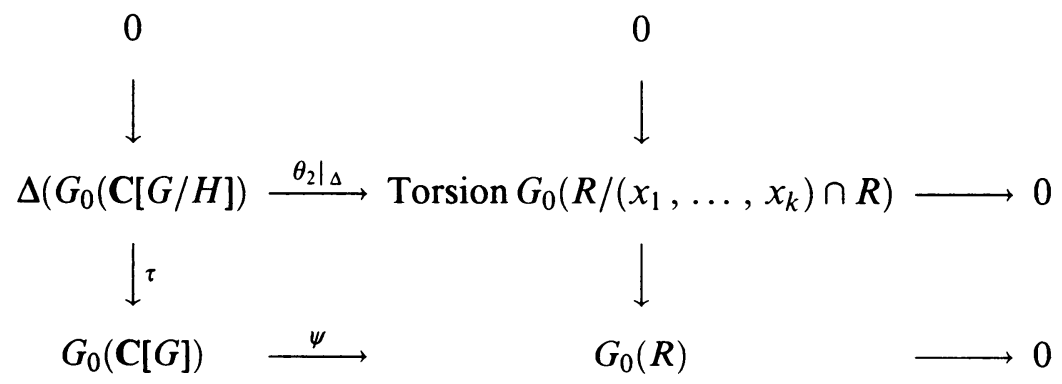

where $\Delta\left(G_{0}(\mathbf{C}[G / H])\right)=\left\{\alpha \in G_{0}(\mathbf{C}[G / H])\right.$ s.t. $\left.\operatorname{dim} \alpha=0\right\}$. 
Now take $\alpha \in \Delta\left(G_{0}(\mathbf{C}[G / H])\right)$. Then

$$
\theta(\alpha)=\theta_{2}(\alpha) \in \text { Torsion } G_{0}\left(R /\left(x_{1}, \ldots, x_{k}\right) \cap R\right)
$$

and therefore $\psi(\tau(\alpha))=0$ if and only if $\theta(\alpha)=0$.

We use these remarks to show that $\operatorname{Ker} \theta_{2}$ is an ideal of $\left.G_{0}(\mathbf{C}[G / H])\right)$.

We have that

$$
\operatorname{Ker} \theta_{2} \subset \Delta\left(G_{0}(\mathbf{C}[G / H])\right) .
$$

Let $\beta \in \operatorname{Ker} \theta_{2}$ and $C$ a $\mathbf{C}[G / H]$-module. Then $\tau([C] \cdot \beta) \in \operatorname{Ker} \psi$, as $\operatorname{Ker} \psi$ is an ideal and $[C] \beta \in \Delta\left(G_{0}(\mathbf{C}[G / H])\right)$ so $\theta_{2}([C \cdot \beta])=0$ i.e. $[C] \beta \in \operatorname{Ker} \theta_{2}$.

The second part of the statement (c) is clear from (b). (c) implies (b).

We have $\operatorname{Ker} \theta=\operatorname{Ker} \theta_{2} \amalg G_{0}\left(C[G] /\left(\underline{e}_{H}\right)\right)$. We want to prove that $\mathbf{Z}[\mathbf{C}[G]] \amalg$ $\left(G_{0}\left(\mathbf{C}[G] /\left(\underline{e}_{H}\right)\right)\right) \amalg \operatorname{Ker} \theta_{2}$ is an ideal of $G_{0}(\mathbf{C}[G])$.

To show this let $J$ be $\mathbf{Z}[\mathbf{C}[G]] \amalg G_{0}\left(\mathbf{C}[G] /\left(\underline{e}_{H}\right)\right) \amalg \operatorname{Ker} \theta_{2}$ and $\alpha, \beta, \gamma$ satisfying $\alpha \in \mathbf{Z}[\mathbf{C}[G]], \beta \in G_{0}\left(\mathbf{C}[G] /\left(\underline{e}_{H}\right)\right)$ and $\gamma \in \operatorname{Ker} \theta_{2}$. If we show that for any $\mathbf{C}[G]$ representation $T$ it is true that $\alpha[T], \beta[T]$ and $\gamma[T]$ are in $J$ we have shown what we wanted. In order to do this we use that for any indecomposable $G$-representation, we have $T^{H}=T$ or $T^{H}=0$, and consider two possibilities for $T$.

(1) If $T^{H}=0$.

Then $\alpha[T] \in \mathbf{Z}[\mathbf{C}[G]], \beta[T] \in \mathbf{Z}[\mathbf{C}[G]] \amalg \operatorname{Ker} \theta$ by hypothesis and that $\gamma[T] \in G_{0}\left(\mathbf{C}[G] / e_{H}\right)$ follows from the fact that $\left(M \otimes_{\mathbf{C}} T\right)^{G}=0$ for any module $M$ with $M^{G}=0$.

(2) If $T^{H}=T$ then $\alpha[T] \in \mathbf{Z}[\mathbf{C}[G]], \beta[T] \in G_{0}\left(\mathbf{C}[G] /\left(e_{H}\right)\right)$ and $\gamma[T] \in$ $\operatorname{Ker} \theta_{2}$, as $\gamma \in \operatorname{Ker} \theta_{2}$ and $\operatorname{Ker} \theta_{2}$ is an ideal of $G_{0}(\mathbf{C}[G / H])$. This finishes the proof.

Corollary 3.5. Suppose $V=V_{1} \amalg_{\mathrm{C}[G]} V_{2}, G$ acts freely on $V_{1} \neq 0$ and faithfully on $V_{2}$. Then we have a commutative diagram

$$
\begin{aligned}
& G_{0}(\mathbf{C}[G]) \stackrel{\theta}{\longrightarrow} G_{0}\left(\mathbf{C}\left[\left[V_{2}\right]\right]^{G}\right) \longrightarrow 0 \\
& \quad \downarrow \sum(-1)^{i}\left[\Lambda^{i} V_{1}\right] \\
& G_{0}(\mathbf{C}[G]) \stackrel{\psi}{\longrightarrow} G_{0}(R) \longrightarrow 0
\end{aligned}
$$

where $\mathbf{C}\left[\left[V_{2}\right]\right]^{G} \cong R /\left(x_{1}, \ldots, x_{k}\right) \cap R$. Furthermore, $\operatorname{Ker} \psi$ is an ideal $\Leftrightarrow \operatorname{Ker} \theta$ is an ideal.

Corollary 3.6. Suppose that $G$ is commutative, $V=V_{1} \amalg_{C[G]} V_{2}$, and that $G$ acts freely on $V_{1}$ and set $H=\operatorname{Ker}\left(G \rightarrow G L\left(V_{2}\right)\right)$. Then $\operatorname{Ker} \psi$ is an ideal if and only if one of the following holds.

(a) $G=H$, or

(b) $\operatorname{Ker} \theta$ is an ideal and $H=1$.

Proof. We only need to prove that the condition is necessary.

If $G \neq H$, Corollary 3.3 shows $H \subset G^{\prime}=\{1\}$, so $H=\{1\}$ and, by Corollary $3.5, \operatorname{Ker} \theta$ is an ideal.

Example 3.7. Let $G$ be the quaternion group. Then $G / G^{\prime}=\mathbf{Z} / 2 \mathbf{Z} \amalg \mathbf{Z} / 2 \mathbf{Z}$. Let $\xi: G \rightarrow G L(2, \mathbf{C})$ be a two dimensional fixed point free representation and $\eta$ 
the representation of $G / G^{\prime}$ given by

$$
\eta(\bar{a}, \bar{b})=\left[\begin{array}{cc}
(-1)^{a} & 0 \\
0 & (-1)^{b}
\end{array}\right], \quad(\bar{a}, \bar{b}) \in \mathbf{Z} / 2 \mathbf{Z} \amalg \mathbf{Z} / 2 \mathbf{Z} .
$$

We view $\eta$ as a representation of $G$ with kernel $G^{\prime}$. Then take the four dimensional representation $\rho=\xi \amalg \eta$ and $R=\left(\mathbf{C}\left[\left[x_{1}, x_{2}, x_{3}, x_{4}\right]\right]\right)^{\rho(G)}$.

With the notations as above, $\operatorname{Ker} \theta_{2}$ is an ideal of $G_{0}\left(\mathbf{C}\left[G / G^{\prime}\right]\right) \cong \mathbf{Z}\left[G / G^{\prime}\right]$. It coincides with the augmentation ideal, and $G_{0}\left(\mathbf{C}[G] /\left(\underline{e}_{G^{\prime}}\right)\right)=\mathbf{Z} \cdot[\xi]$. Since $\xi \otimes_{\mathbf{C}} \xi=\mathbf{C}[G]=2 \xi \in \mathbf{Z}[\mathbf{C}[G]] \amalg \operatorname{Ker} \theta$, we get that $\operatorname{Ker}\left(G_{0}(\mathbf{C}[G]) \rightarrow G_{0}(R)\right)$ is an ideal.

We remark that in this example $G_{0}\left(\mathbf{C}[G] / \underline{e}_{G^{\prime}}\right)$ is not an ideal.

\section{ONE RAMIFIED LINE}

In this section we consider the following particular case of $\S 3: G$ acts on $V=V_{1} \amalg_{\mathbf{C}[G]} V_{2}$ (without pseudo-reflections) with $V_{1}=\mathbf{C} x_{1} \amalg_{\mathbf{C}} \cdots \amalg_{\mathbf{C}} \mathbf{C} x_{n-1}$ and $V_{2}=\mathbf{C} x_{n}$. We assume that $G$ acts freely on $V_{1}$ and that the ramification locus consists of the line $\mathbf{C} x_{n}$.

Theorem 4.1. Let $\psi: G_{0}(\mathbf{C}[G]) \rightarrow G_{0}(R)$ be given by $\psi([W])=\left[\left(S \otimes_{\mathbf{C}} W\right)^{G}\right]$. Then $\operatorname{Ker} \psi$ is an ideal if and only if $G$ acts trivially on $V_{2}$.

Proof. First we show that we can assume $\operatorname{Ker}\left(G \rightarrow G L\left(\mathbf{C} x_{n}\right)\right)=G^{\prime}$.

Let $a$ denote the element $a=\sum(-1)^{i}\left[\Lambda^{i} V_{1}\right]$ of $G_{0}(\mathbf{C}[G])$. Since $G$ acts freely on $V_{1}$ we have

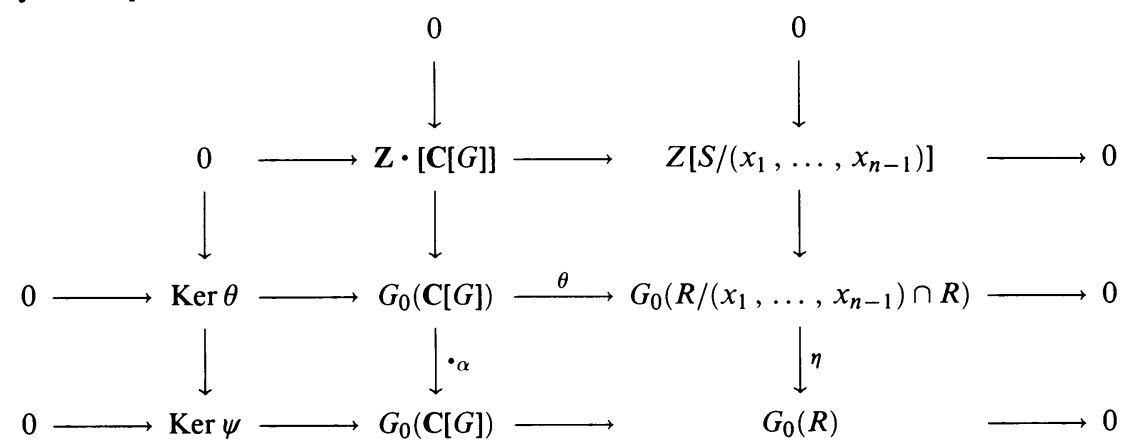

We will show that $\eta=0$ which is equivalent to $G$ acting trivially on $\mathbf{C} x_{n}$.

We have that $\operatorname{Ker}\left(G \rightarrow G L\left(\mathbf{C} x_{n}\right)\right) \supset G^{\prime}$. If $\operatorname{Ker}\left(G \rightarrow G L\left(\mathbf{C} x_{n}\right)\right) \neq G^{\prime}$ then for some one dimensional representation $Z$ we have $a[Z] \in \operatorname{Ker} \psi$ and it follows that $\left(a[Z] \cdot\left[Z^{*}\right]\right)=a \in \operatorname{Ker} \psi$. Then $\eta=0$.

We can hence assume $\operatorname{Ker}\left(G \rightarrow G L\left(\mathbf{C} x_{n}\right)\right)=G^{\prime}$, in which case $G / G^{\prime}$ is cyclic. If $k a \in \operatorname{Ker} \psi$, then $k\left[R /\left(x_{1}, \ldots, x_{n-1}\right) \cap R\right]=0$ in $G_{0}(R)$, and so $k|| G / G^{\prime} \mid$.

If now $W_{1}$ and $W_{2}$ are indecomposable $\mathbf{C}[G]$-modules and $Z$ is a one dimensional $\mathbf{C}[G]$-module, then the multiplicity of $Z$ in $W_{1} \otimes_{\mathbf{C}} W_{2}$ is less than or equal to one.

Hence, if $W$ is an indecomposable representation of dimension bigger than one, we have

$$
\left[W \otimes W^{*}\right]=\sum_{\substack{\operatorname{dim} W_{i}>1 \\ W_{i} \text { indec }}} \lambda_{W_{i}}\left[W_{i}\right]+\sum_{\substack{j=1 \\ \operatorname{dim} Z_{j}=1}}^{\left|G / G^{\prime}\right|} u_{j}\left[Z_{j}\right]
$$


Now suppose that $G$ is a counterexample for our theorem. We claim that $\operatorname{dim} W>1$ implies that all the $u_{j}$ in the formula above equal one. For

$$
\begin{aligned}
& a\left(\sum_{\operatorname{dim} W_{i}>1} \lambda_{W_{i}}\left[W_{i}\right]+\sum_{j=1}^{\left|G / G^{\prime}\right|} u_{j}\left[Z_{j}\right]\right) \\
& \quad=a\left(\sum_{\operatorname{dim} W_{i}>1} \lambda_{W_{i}}\left[W_{i}\right]+\sum_{j=1}^{\left|G / G^{\prime}\right|} u_{j}\left(Z_{j}-1\right)+\sum_{j=1}^{\left|G / G^{\prime}\right|} u_{j}\right) \in \operatorname{Ker} \psi,
\end{aligned}
$$

and by the fundamental diagram $\operatorname{Ker} \psi$ is generated by

$$
\left\{a\left[W_{i}\right] \text {, s.t. } \operatorname{dim} W_{i}>1 ; a\left(Z_{j}-1\right) \text { s.t. } \operatorname{dim} Z_{j}=1\right\} .
$$

If it is an ideal, we get $a\left(\sum u_{j}\right) \in \operatorname{Ker} \psi$. Since the coefficient of $1_{\mathbf{C}}$ in $[W \otimes$ $\left.W^{*}\right]$ is one it follows that all $u_{j}$ equal one.

So $W \otimes Z \cong W$ for every $Z$ of dimension one and $W$ indecomposable with $\operatorname{dim} W>1$. If then $\chi_{W}$ is the character associated to $W$, we have $\chi_{W}(g)=0$ for all $g \notin G^{\prime}$, as $\chi_{W}(g) \rho(g)=\chi_{W}(g)$ for every linear character $\rho$.

Let $\left(\chi_{1}, \ldots, \chi_{t}\right)$ be all the irreducible characters of $G$. Then by the second relation of orthogonality we get $\sum_{m=1}^{t} \chi_{m}(g) \overline{\chi_{m}}(g)=\left|C_{G}(g)\right|$, (the order of the centralizer of $g$ ). If $g \notin G^{\prime}$, we get from above that $\left|C_{G}(g)\right|=\left|G / G^{\prime}\right|$. Now take $x$ in $G$ s.t. $\bar{x}$ generates $G / G^{\prime}$. As $\langle x\rangle \subset C_{G}(x)$, we have $\langle x\rangle \cap G^{\prime}=\{1\}$. Now take $y \in\langle x\rangle$ with $|y|=p, p$ prime, $p>1$. Then it follows that $C_{G}(y)=$ $\langle x\rangle$. Next, take $z \in G^{\prime}$ s.t. $|z|=q, q$ prime, $q>1$. Then $|\langle y, z\rangle|=$ p.q., as $\langle y, z\rangle$ acts freely on $V_{1}$. It follows from [17, Theorem 5.8.1] that $\langle y, z\rangle$ is cyclic, so $z \in C_{G}(y)$ and hence $z \in\langle x\rangle$, a contradiction.

In view of the preceding theorem, the following question arises naturally. Assume $V=V_{1} \amalg V_{2}, G$ acts freely on $V_{1}$ and $a=\sum_{i=1}^{\operatorname{dim} V}\left[\Lambda^{i} V_{1}\right]$. Under what conditions is it true that the ideal generated by $\operatorname{Ker} \psi$ is $a \cdot G_{0}(\mathbf{C}[G])$ ? In case that $V_{2}$ is a line, we have the following answer.

Proposition 4.2. Let $V=V_{1} \amalg_{\mathbf{C}[G]} V_{2}$, with $V_{1}=\mathbf{C} x_{1} \amalg_{\mathbf{C}} \cdots \amalg_{\mathbf{C}} \mathbf{C} x_{n-1}, V_{2}=\mathbf{C} x_{n}$, and set $H=\operatorname{Ker}\left(G \rightarrow G L\left(V_{2}\right)\right)$. Suppose that $G$ acts freely on $V_{1}$, with $\operatorname{dim} V_{1}>1$ and set $d=\operatorname{gcd}\left\{\operatorname{dim}_{\mathrm{C}} W\right.$ s.t. $\left.W \in \operatorname{Ind}\left(\mathbf{C}[G] /\left(\underline{e}_{H}\right)\right)\right\}$.

Let $\underline{I}$ be the ideal generated by $\operatorname{Ker}\left(G_{0}(\mathbf{C}[G]) \rightarrow G_{0}(R)\right)$ and

$$
a=\sum_{i=0}^{\operatorname{dim} V_{1}}(-1)^{i}\left[\Lambda^{i} V_{1}\right]
$$

Then $\underline{I}=(\underline{a})=a G_{0}(C[G])$ if and only if $d=1$.

Proof. Using the fundamental diagram, it follows that $(\underline{a})=\underline{I} \Leftrightarrow a \in I$. If $d=1$, then by similar arguments as before, $\underline{a} \in \underline{I}$.

Suppose $a \in \underline{I}$. If $H \neq G^{\prime}$ then $d=1$. So suppose $H=G^{\prime}$. Then $\underline{I}$ is the ideal generated by the set

$\{a W: W$ indecomposable and $\operatorname{dim} W>1, a(Z-1)$ s.t. $\operatorname{dim} Z=1\}$.

Since

$$
a=\sum_{\substack{\operatorname{dim} W>1 \\ W \text { indec }}} \lambda_{W} a W+\sum_{\operatorname{dim} Z=1} u_{Z} a(Z-1)
$$

and both $\lambda_{W}$ and $u_{Z} \in G_{0}(C[G])$. 
Therefore

$$
a\left(1-\sum_{\substack{\operatorname{dim} W>1 \\ W \text { indec }}} \lambda_{W} W-\sum_{\operatorname{dim} Z=1} u_{Z}(Z-1)\right)=1,
$$

by Lemma 1.2. As $\operatorname{ann}(\underline{a})=\mathbf{Z} \cdot[\mathbf{C}[G]]$, it follows

$$
1-\sum_{\operatorname{dim} W=1} \lambda_{W} W-\sum_{\operatorname{dim} Z=1} u_{Z}(Z-1)=m[\mathbf{C}[G]]
$$

for some $m \in \mathbf{Z}$. Consequently, $m|G|=\sum_{\operatorname{dim} W>1}\left(\operatorname{dim} \lambda_{W}\right)(\operatorname{dim} W)-1$. Since $d \mid m$ and $d \mid \operatorname{dim} W$, it follows that $d \mid 1$, so $d=1$.

Example 4.3. Let $G$ be the binary dihedral group $D_{3}^{*}$.

$$
D_{3}^{*}=\left\langle A, B: A^{3}=B^{4}=1, B A B^{-1}=A^{-1}\right\rangle, \quad D_{3}^{*} /\left(D_{3}^{*}\right)^{\prime}=D_{3}^{*} /\langle A\rangle \cong \mathbf{Z} / 2 \mathbf{Z} .
$$

It has 2 one-dimensional representations and 2 two-dimensional representations. Let $\Gamma$ be the following representation

$$
\begin{array}{cc}
\Gamma(A)=\left[\begin{array}{ccc}
W & 0 & 0 \\
0 & W^{2} & 0 \\
0 & 0 & 1
\end{array}\right] ; & W=e^{2 \pi i / 3}, \\
\Gamma(B)=\left[\begin{array}{ccc}
0 & 1 & 0 \\
-1 & 0 & 0 \\
0 & 0 & i
\end{array}\right] ; & i^{2}=-1 .
\end{array}
$$

Then we are in the situation above and the number $d$ in the Proposition 4.2 is 2, so $I \neq(\underline{a})$.

\section{FORMAL POWER SERIES}

In this section we prove that for any quotient singularity $R$,

$$
G_{0}(R) \cong G_{0}(R[[t]]),
$$

where $R[[t]]$ is the ring of formal series in the variable $t$, with coefficients from $R$.

This fact is a consequence of the following.

Proposition 5.1. Suppose $U$ is a commutative complete ring. Suppose further that there is a module $T$ in mod-U s.t. $G_{0}(U[[t]])$ is generated by the classes of objects in $\operatorname{add}\left(T \otimes_{U} U[[t]]\right)$. Then the functor from $\bmod U$ to $\bmod U[[t]]$ given by $N \rightarrow N \otimes_{U} U[[t]]$ induces an isomorphism $\varphi: G_{0}(U) \rightarrow G_{0}(U[[t]])$.

Proof. Let $T[[t]]=A_{1} \amalg_{U[[t]]} \cdots \amalg_{U[[t]]} A_{n}$ with $A_{i}$ indecomposable. Then $T[[t]] \cong T \otimes U[[t]] \cong \coprod_{i=1}^{n}\left(A_{i} / t A_{i}\right) \otimes U[[t]]$ and $A_{i} / t A_{i} \neq 0$ because $t \in$ $\operatorname{rad} U[[t]]$.

Since $U[[t]]$ is complete by the Krull-Schmidt Theorem, it follows that $A_{i} / t A_{i}$ is indecomposable for every $i$ and that for each $i$ there is a $j$ s.t. $A_{i} \cong A_{j} / t A_{j} \otimes_{U} U[[t]]$. Then every [ $\left.A_{i}\right]$ is in the image of $\varphi$. But by hypothesis the elements $\left[A_{i}\right]$ generate $G_{0}(U[[t]])$, whence it follows that $\varphi$ is onto. 
Since it holds for any ring $U$ that the map $\varphi$ is a split monomorphism, it follows that $\varphi$ is an isomorphism.

Theorem 5.2. Let $R=S^{G}$ be a quotient singularity. Then $G_{0}(R) \cong G_{0}(R[[t]])$. Proof. Since $|G|^{-1} \in R$, we know that the fixed point functor from $S[G]$-mod to $R$-mod is exact and dense. Hence it induces an epimorphism $G_{0}(S[G]) \rightarrow$ $G_{0}(R)$.

$G_{0}(S[G])$ is generated by the classes of direct summands of $S$. Now we extend the action of $G$ to $S[[t]]$, letting $G$ act trivially on $t$. Then $(S[[t]])^{G} \cong$ $R[[t]]$ and $G_{0}(R[[t]])$ is generated by the classes of summands of $S[[t]] \cong$ $S \otimes_{R} R[[t]]$. So by Proposition 5.1 the map $\varphi: G_{0}(R) \rightarrow G_{0}(R[[t]])$ is an isomorphism.

Counterexample 5.5. The ring $U=Q(u)[[x, y, z]] /\left(y^{2}+x^{3}+u z^{6}\right)$, where $Q(u)$ is the field of fractions of $Q[u]$, is factorial, see [11]. It is easy to prove that $[Q(u)]=0$ in $G_{0}(U)$. Since $U$ has dimension two, it follows that $G_{0}(U) \cong$ $\mathbf{Z}[U]$. But $\mathrm{Cl}(U[[t]])$ is not torsion, so rank $G_{0}(U[[t]])>1$. Thus there is no isomorphism between $G_{0}(U)$ and $G_{0}(U[[t]])$.

Remarks. (1) We do not know any counterexample where $U$ has a field of representatives which is algebraically closed.

(2) The fact that $G_{0}(U) \cong G_{0}(U[t])$, see [6], shows an interesting difference in the behavior of the polynomial ring $U[t]$ and the formal power series $U[[t]]$.

\section{Chapter III. Some 3-Dimensional cases}

In this chapter we are going to compute some cases of Grothendieck groups of three dimensional quotient singularities. We will analyze some related questions, for example:

(a) When is $\operatorname{Ker}\left(G_{0}(S[G]) \rightarrow G_{0}(R)\right)$ an ideal?

(b) When is $G_{0}(R)$ generated by the classes $[M]$ of $R$-modules $M$ that are free outside the maximal ideal?

We remark that the subgroup of $G_{0}(R)$ generated by the classes of $R$-modules $M$ that are free outside maximal ideals is a subring of the Grothendieck ring. (See $\S 2$, Chapter II for definitions.) We denote it by $\mathscr{A}(R)$.

Proposition 1.1. Suppose $R=S^{G}=C[[V]]^{G} ; G \subset G L(V)$ without pseudoreflections. If $G$ is commutative, then $G_{0}(R)=\mathscr{A}(R)$ if and only if $G$ acts freely on $V$.

Proof. Suppose $G$ does not act freely and let $p$ be a nonmaximal prime which is ramified. Clearly, if $G_{0}(R)=\mathscr{A}(T)$, then $G_{0}\left(R_{\underline{p}}\right)=\mathrm{Z} \cdot\left[R_{\underline{p}}\right]$, so $R_{\underline{p}}$ is a U.F.D. But as $G$ is commutative, $S_{\underline{p}}$ can be decomposed as a direct sum of rank one reflexive modules. free.

Since $R_{\underline{p}}$ is a U.F.D. every rank one reflexive module is free, so $S_{\underline{p}}$ is $R_{\underline{p}}$

Then, by the theorem on the purity of branch locus, $R_{\underline{p}}$ is regular, a contradiction.

From now on we assume that $G \subset G L(3, C)$ is finite and that it contains no pseudo-reflection; set $S=C\left[\left[x_{1}, x_{2}, x_{3}\right]\right]$ and $R=S^{G}$. 
Proposition 1.2. Suppose $G$ does not act freely on $V$ and let $\left\{\underline{p}_{1}, \ldots, \underline{p}_{n}\right\}$ be the nonmaximal ramified primes of $R$. With $T=\left(\bigcup_{i=1}^{m} \underline{p}_{i}\right)$ one has $G_{0}(R) / \mathscr{A}(R)$ $\cong G_{0}\left(R T^{-1}\right) / \mathbf{Z} \cdot\left[R T^{-1}\right]$.

Proof. We have a structure of $\mathscr{A}(R)$ - mod on $G_{0}\left(R T^{-1}\right)$ given by $[A] \alpha$ $=(\operatorname{rank} A) \alpha$ for $A$ an $R$-module, free outside the maximal ideal and, $\alpha \in$ $G_{0}\left(R T^{-1}\right)$.

Since $\left[\left(A \otimes_{R} M\right) T^{-1}\right]=(\operatorname{rank} A) \cdot\left[M T^{-1}\right]$, for $A$ free outside the maximal ideal and $M$ any $R$-module, we get that the natural map $\varphi: G_{0} \rightarrow$ $G_{0}\left(R T^{-1}\right)$ is an $\mathscr{A}(R)$-module homomorphism.

Let $\underline{D}$ be the subcategory of modules $M$ s.t. $M T^{-1}=0$. Then if $M \in \underline{\underline{D}}$ we take $0 \rightarrow \Omega^{3} M \rightarrow F_{3} \rightarrow F_{2} \rightarrow F_{1} \rightarrow M \rightarrow 0$ exact with $F_{i}$ free and finitely generated, so that $\Omega^{3} M$ is a Cohen-Macaulay $R$-module. The fact that $\left(\Omega^{3} M\right) T^{-1}$ is free implies that $\left[\Omega^{3} M\right]$ is in $\mathscr{A}(R)$. We get that

$$
\operatorname{Ker}\left(G_{0}(R) \rightarrow G_{0}\left(R T^{-1}\right)\right) \subset \mathscr{A}(R),
$$

hence

$$
G_{0}(R) / \varphi^{-1}\left(\mathbf{Z}\left[R T^{-1}\right]\right) \cong G_{0}\left(R T^{-1}\right) / \mathbf{Z}\left[R T^{-1}\right]
$$

and

$$
\varphi^{-1}\left(\mathbf{Z}\left[R T^{-1}\right]\right)=\mathbf{Z}[R] \amalg \operatorname{Ker} \varphi \subset \mathscr{A}(R) .
$$

Since $\mathbf{Z}(R) \subset \varphi^{-1}\left(\mathbf{Z}\left[R T^{-1}\right]\right)$ we get $\mathscr{A}(R)=\varphi^{-1}\left(\mathbf{Z}\left[R T^{-1}\right]\right)$ and

$$
G_{0}\left(R T^{-1}\right) / \mathbf{Z}\left[R T^{-1}\right] \cong G_{0}(R) / \mathscr{A}(R) \cong \operatorname{Torsion}\left(G_{0}\left(R T^{-1}\right)\right) .
$$

Corollary 1.3. With the hypotheses of Theorem 1.2, the following three conditions are equivalent:

(1) $G_{0}(R)=\mathscr{A}(R)$.

(2) $G_{0}\left(R T^{-1}\right) \cong \mathbf{Z}$.

(3) $R T^{-1}$ is a U.F.D. and $\left[\left(R / \underline{p}_{i}\right) T^{-1}\right]=0$ in $G_{0}\left(R T^{-1}\right)$ for $\underline{p}_{i}$ a nonramified prime ideal.

1.4. We finish the paper by looking at three special cases in dimension three. Case 1. Suppose $V=\left(\mathbf{C} x_{1} \amalg_{\mathbf{C}} \mathbf{C} x_{2}\right) \amalg_{\mathbf{C}[G]} \mathbf{C} x_{3}$ and the ramification locus consists of the line $\mathbf{C} x_{3}$.

Then one has the exact sequence

$$
0 \rightarrow G_{0}\left(R /\left(x_{1}, x_{2}\right) \cap R\right) / \mathbf{Z}\left[S /\left(x_{1}, x_{2}\right)\right] \rightarrow G_{0}(R) \rightarrow G_{0}\left(R /\left(x_{3}\right) \cap R\right) \rightarrow 0
$$

and $G_{0}\left(R /\left(x_{3}\right) \cap R\right) \cong \mathrm{Z} \amalg \mathrm{Cl}(R) \cong \mathbf{Z} \amalg G^{*}$.

We know (Theorem 5.1 of Chapter II) that $\operatorname{Ker}\left(G_{0}(S[G]) \rightarrow G_{0}(R)\right)$ is an ideal if and only if $G$ acts trivially on $\mathbf{C} x_{3}$.

We have, in this case, a characterization of $\mathscr{A}(R)=G_{0}(R)$. We have shown (Proposition 1.2) that $\mathscr{A}(R)$ is generated by the classes [M], of $R$-modules $M$, s.t. $M_{\underline{p}}$ is free, where $\underline{p}=\left(x_{1}, x_{2}\right) \cap R$.

Claim. $\left[R /\left(\left(x_{1}, x_{2}\right) \cap R\right)\right]_{\underline{p}}=0$ in $G_{0}\left(R_{\underline{p}}\right)$.

Proof. To begin with, assume that $G$ is cyclic. Then we can suppose that $G$ acts diagonally on $V$ and that $\mathbf{C}\left[\left[x_{2}, x_{3}\right]\right] \cong S /\left(x_{1}\right)$ is a $G$-module. As $\left[\left(S /\left(x_{1}\right)\right)\right]^{G}=R /\left(x_{1}\right) \cap R$ is an integrally closed domain, we see that

$$
R /\left(\left(x_{1}\right) \cap R\right) \otimes R_{\underline{p}}=\left(R /\left(x_{1}\right) \cap R\right)_{\underline{p}}
$$


is a DVR. Let $t$ be a generator of the maximal ideal. We have

$$
0 \rightarrow\left(\frac{R}{\left(x_{1}\right) \cap R}\right)_{\underline{p}}^{\stackrel{\bullet}{\rightarrow}}\left(\frac{R}{\left(x_{1}\right) \cap R}\right)_{\underline{p}} \rightarrow\left(\frac{R}{\left(x_{1}, x_{2}\right) \cap R}\right)_{\underline{p}} \rightarrow 0
$$

which shows that $\left[\left(R /\left(x_{1}, x_{2}\right) \cap R\right)_{p}\right]=0$ in $G_{0}\left(R T^{-1}\right)$.

If $G$ is not cyclic, set $H=\operatorname{Ker}\left(\bar{G} \rightarrow G L\left(\mathbf{C} x_{3}\right)\right)$ and take $\sigma$ s.t. $\bar{\sigma}$ generates $G / H$. Then

$$
\left[\left(\frac{S}{\left(x_{1}, x_{2}\right)}\right)^{G}\right]=\left[\left(\frac{S}{\left(x_{1}, x_{2}\right)}\right)^{G / H}\right]=\left[\frac{R}{\left(x_{1}, x_{2}\right) \cap R}\right]=0
$$

in $G_{0}\left(C[[V]]^{\langle\sigma\rangle}\right)$, so $\left[\left(S /\left(x_{1}, x_{2}\right)\right)^{G}\right]=0$ in $G_{0}(R)$.

As $G_{0}(R)=\mathscr{A}(R)$ if and only if $R_{\underline{p}}$ is a U.F.D., we need that $\left(S \otimes_{\mathrm{C}} W_{i}\right)_{\underline{p}}^{G} \cong$ $R_{\underline{p}}$ for every $\mathbf{C}[G]$-module $W_{i}$ with $\overline{\operatorname{dim}} W_{i}=1$.

We have ring homomorphisms $\mathbf{C}\left(\left(x_{3}\right)\right)[G] \stackrel{i}{\rightarrow} S_{p}[G] \stackrel{\pi}{\rightarrow}\left(S_{p} / p S_{p}\right)[G] \cong$

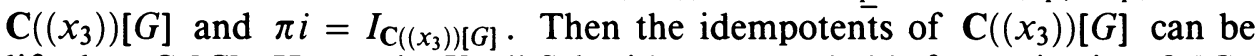
lifted to $S_{\underline{p}}[G]$. Hence the Krull-Schmidt property holds for projective $S_{\underline{p}}[G]-$ modules. It follows that the modules of the form $S \otimes_{\mathbf{C}} W_{i}$ with $\operatorname{dim} W_{i}=1$, for which $\left(S \otimes_{\mathbf{C}} W_{i}\right)^{G} \cong R_{\underline{p}}$, are exactly the ones with $W_{i} \in \mathbf{C}[G / H]$. Then one sees that for $R_{\underline{p}}$ to be a U.F.D. it is necessary and sufficient that $H=G^{\prime}=[G, G]$.

Remarks. (1) There are various examples where $G \subset G L(2, C)$ contains no pseudo-reflections and $G / G^{\prime}$ is cyclic, see [9]. Using these, we construct examples where $\operatorname{Ker}\left(G_{0}(\mathbf{C}[G]) \rightarrow G_{0}(R)\right)$ is not an ideal but $\mathscr{A}(R)=G_{0}(R)$.

(2) If $G$ acts trivially on $\mathbf{C} x_{3}$ then $\operatorname{Ker}\left(G_{0}(\mathbf{C}[G]) \rightarrow G_{0}(R)\right)$ is an ideal and $\mathscr{A}(R)=\mathbf{Z} \cdot[R]$.

Case 2. Suppose that the span of the ramification locus has dimension two.

The span of the ramification locus is a $\mathbf{C}[G]$-module so in this case $V=$ $V_{1} \amalg_{\mathrm{C}[G]} V_{2}$, where $V_{2}$ is the span of the ramification locus, that has dimension two, and $\operatorname{dim} V_{1}=1$. Moreover $G$ acts freely on $V_{1}$ which implies that $G$ is cyclic. To continue our discussion we need the following lemma, which is easy to prove.

Lemma 1.5. Let $G$ be a commutative group acting on a three dimensional vector space without pseudo-reflections. If

$$
V=\mathbf{C} x_{1} \amalg_{\mathbf{C}[G]} \mathbf{C} x_{2} \amalg_{\mathbf{C}[G]} \mathbf{C} x_{3},
$$

then the ramification locus is contained in $\bigcup_{i=1}^{3} \mathbf{C} x_{1}$. Therefore if the span of ramification locus has dimension two, we can assume $V=C x_{1} \amalg_{C[G]} \mathbf{C} x_{2} \amalg_{\mathbf{C}[G]} \mathbf{C} x_{3}$ with ramification locus equal to $\mathbf{C} x_{2} \cup \mathbf{C} x_{3}$.

We describe $G_{0}(R)$ in this case. Since $G\left(R /\left(x_{2}, x_{3}\right) \cap R\right)=\mathbf{Z}$, Theorem 1.1 of Chapter II yields the exact sequence

$$
0 \rightarrow G_{0}\left(R /\left(x_{1}\right) \cap R\right) / \mathbf{Z}\left[S /\left(x_{1}\right)\right] \rightarrow G_{0}(R) \rightarrow \mathbf{Z} \rightarrow 0 .
$$

This shows that Torsion $G_{0}(R)=\operatorname{Im}\left(G_{0}\left(R /\left(x_{1}\right) \cap R\right) \rightarrow G_{0}(R)\right)$.

Suppose $G=\left\langle\sigma: \sigma^{m}=1\right\rangle$ and that the representation associated with $V$ is given by

$$
\sigma \rightarrow\left[\begin{array}{ccc}
\xi & 0 & 0 \\
0 & \xi^{k_{1}} & 0 \\
0 & 0 & \xi^{k_{2}}
\end{array}\right] \text { where } \xi \text { is a primitive } m \text { th root of unity. }
$$


If we want no pseudo-reflections, we need $\left(\left(k_{1}, k_{2}\right), m\right)=1$, where $(a, b)$ denotes the greatest common divisor of $a$ and $b$.

Let $K$ be the subgroup generated by $\sigma^{m /\left(m, k_{1}\right)}$ and $\sigma^{m /\left(m, k_{2}\right)}$. Then $K=$ $\left\langle\sigma^{a}\right\rangle$ where

$$
a=m /\left(\left(m, k_{1}\right) \cdot\left(m, k_{2}\right)\right)
$$

and $|G / K|=a$. Moreover $\operatorname{Im}\left(K \rightarrow G L\left(\mathbf{C} x_{2} \amalg \mathbf{C} x_{3}\right)\right)$ is the subgroup of $G$ generated by pseudo-reflections with respect to the representation

$$
\sigma \rightarrow\left[\begin{array}{cc}
\xi^{k_{1}} & 0 \\
0 & \xi^{k_{2}}
\end{array}\right]
$$

We have the isomorphisms

$$
G_{0}\left(R /\left(x_{1} \cap R\right)\right) \cong \mathbf{Z}\left[R /\left(x_{1}\right) \cap R\right] \amalg(G / K)^{*} \cong \mathbf{Z} \amalg(G / K) .
$$

We have the isomorphism

$$
S /\left(x_{1}\right)=(m / a)\left(S /\left(x_{1}\right)\right)^{(G / K)} \cong(m / a) \coprod_{i=1}^{a}\left(S /\left(x_{1}\right) \otimes W_{i}\right)^{G / K},
$$

where $T=\left\{W_{i}\right\}$ is the set of indecomposable $\mathbf{C}[G / k]$ representations. So using the isomorphism $(* *)$ we obtain

$$
\left[S /\left(x_{1}\right)\right] \rightarrow(m / a)\left(a, \sum_{\alpha_{i} \in T} \alpha_{i}\right)=\left(m,(m / a) \sum_{\alpha_{i} \in T} \alpha_{i}\right) .
$$

It follows that if $m / a$ is even or $a$ is odd and $(m / a) \sum_{\alpha_{i} \in T} \alpha_{i}=0$, in which case

$$
G_{0}\left(R /\left(x_{1}\right) \cap R\right) / Z\left[S /\left(x_{1}\right)\right] \cong G^{*} \amalg(G / K)^{*}=\left\langle\left[\left(S /\left(x_{1}\right)\right)^{G}\right]\right\rangle \amalg\left\langle\left[S /\left(x_{1}, x_{2}\right)^{G}\right]\right\rangle .
$$

If $m / a$ is odd and $a$ is even then in the cyclic group $(G / K)^{*}$ there is exactly one element of order 2 , let us say $\alpha_{1}$, and via the isomorphism we have $(m / a)\left(\sum_{\alpha_{i} \in T} \alpha_{i}\right)=\alpha_{1}$ and $\left[S /\left(x_{1}\right)\right] \rightarrow\left(m, \alpha_{1}\right)$ with $2\left[S /\left(x_{1}\right)\right]=(2 m, 0)$. So $\left[\left(S /\left(x_{1}\right)\right)^{G}\right]$ is an element of order $2 m$ of $G_{0}(R)$ and

$$
\left\langle\left(S /\left(m_{1}\right)\right)^{G}\right\rangle \cap\left\langle\left(S /\left(x_{1}, x_{2}\right)\right)^{G}\right\rangle
$$

is a group of order two.

Torsion $G_{0}(R)$ has exponent $2 m$. So there is an epimorphism $\pi: G_{0}(R) \rightarrow$ $\mathbf{Z} /(a / 2) \mathbf{Z}$ whose kernel is the subgroup generated by $\left(S /\left(x_{1}\right)\right)^{G}$. If we take the element $t=2 m / a\left[\left(S /\left(x_{1}\right)\right)^{G}\right]-\left[\left(S /\left(x_{1}, x_{2}\right)\right)^{G}\right]$ then

$$
\text { Torsion } G_{0}(R)=\left\langle\left[\left(S /\left(x_{1}\right)\right)^{G}\right]\right\rangle \amalg\left\langle 2 m / a\left[\left(S /\left(x_{1}\right)\right)^{G}\right]-\left[\left(S /\left(x_{1}, x_{2}\right)\right)^{G}\right]\right\rangle .
$$

Another question that we want to answer is: When is $\operatorname{Ker}\left(G_{0}(\mathbf{C}[G]) \rightarrow G_{0}(R)\right)$ an ideal? By Corollary 3.5 of Chapter II this is equivalent with $\operatorname{Ker} \theta$ being an ideal. One can prove that this happens iff $K=G$ which happens if and only if the action of $G$ on $\mathbf{C} x_{2} \amalg \mathbf{C} x_{3}$ is generated by pseudo-reflections.

Case 3. We finally look at the following special case. $G$ is a cyclic group acting on $V=\mathbf{C} x_{1} \amalg_{\mathbf{C}[G]} \mathbf{C} x_{2} \amalg_{\mathbf{C}[G]} \mathbf{C} x_{3}$ with span of the ramification locus equal to $V$. We see by Lemma 1.5 that the ramification locus is $\bigcup_{i=1}^{3} \mathbf{C} x_{i}$. 
One can show that a cyclic group has a three dimensional representation with these properties if and only if there is $H \subset G$ with $H=H_{1} \times H_{2} \times H_{3}$ and $H_{i}=\operatorname{Ker}\left(G \rightarrow G L\left(C x_{i}\right)\right), H_{i} \neq 0$.

We identify $G_{0}(\mathbf{C}[G])$ with the ring of characters of $G$ and we want to describe $\operatorname{Ker}\left(G_{0}(\mathbf{C}[G]) \rightarrow G_{0}(R)\right)$. Let $\chi_{i}$ be the character associated with $G \rightarrow G L\left(\mathbf{C} x_{i}\right)$ and $\chi=\chi_{1}+\chi_{2}+\chi_{3}$ the character associated with $V$. Let $\underline{b}=\bigcap_{i \neq j}\left(x_{i}, x_{j}\right)$ which is an $S[G]$-submodule of $S$. Then we have

0

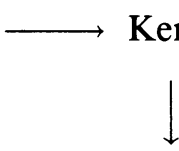

$0 \longrightarrow \operatorname{Ker} \psi$
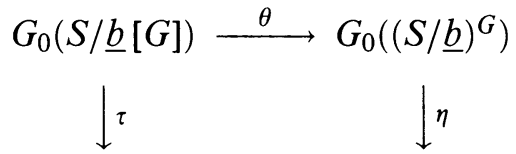

$G_{0}(S[G])$
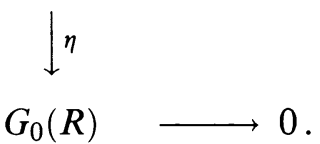

Take $t \in m_{R} \backslash \underline{b}$ to obtain a commutative exact diagram

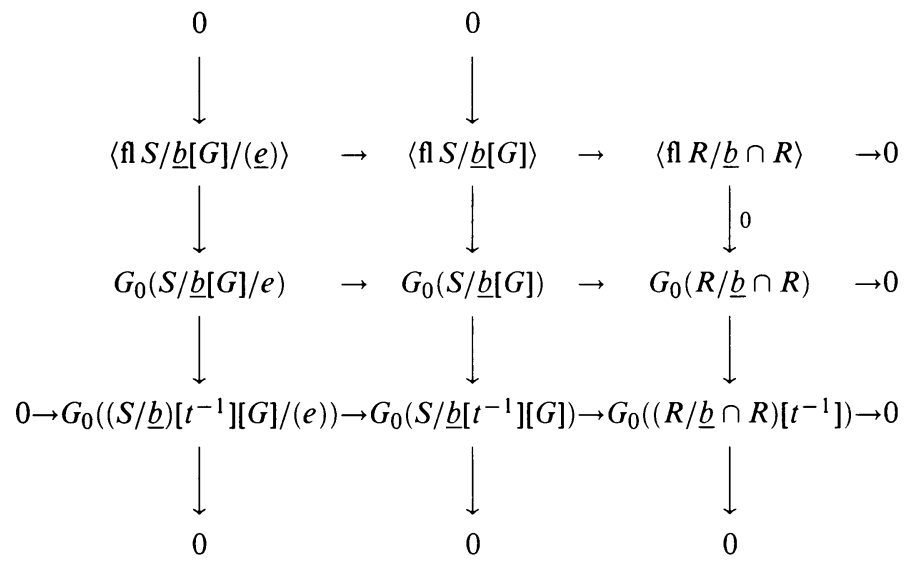

where $\left\langle\mathrm{fl} G_{0}(-)\right\rangle$ means the subgroup of $G_{0}(-)$ generated by the classes of finite length modules. We can show then that $\operatorname{Ker} \psi$ is the subgroup generated by the following set:

$$
\begin{aligned}
& \left\{\amalg_{i=1}^{3}\left(i-\chi_{i}\right) G_{0}(\mathbf{C}[G]),\right. \\
& \left(1-\chi_{2}\right)\left(1-\chi_{3}\right) \chi_{j_{1}}: \chi_{j_{1}} \in \operatorname{Ind}\left(\mathbf{C}[G] /\left(\underline{e}_{H_{1}}\right)\right), \\
& \left(1-\chi_{1}\right)\left(1-\chi_{3}\right) \chi_{j_{2}}: \chi_{j_{2}} \in \operatorname{Ind}\left(\mathbf{C}[G] /\left(\underline{e}_{H_{2}}\right)\right), \\
& \left.\left(1-\chi_{1}\right)\left(1-\chi_{2}\right) \chi_{j_{3}}: \chi_{j_{3}} \in \operatorname{Ind}\left(\mathbf{C}[G] /\left(\underline{e}_{H_{3}}\right)\right)\right\}
\end{aligned}
$$

where

$\chi_{i}$ is the representation associated with $G \rightarrow G L\left(\mathbf{C}\left(x_{i}\right)\right)$,

$\mathscr{H}_{i}=\operatorname{Ker} \chi_{i}$ and $\chi=\chi_{1}+\chi_{2}+\chi_{3}$ is associated with $V$.

We have that $\operatorname{Ker} \psi$ is an ideal if and only if $\operatorname{Ker} \psi=\operatorname{Im} \tau$ in diagram (1), or equivalently $\eta=0$.

This is the case if $H=G$, where $H=H_{1} \times H_{2} \times H_{3}$ with $H_{i}=$ $\operatorname{Ker}\left(G \rightarrow G L\left(\mathbf{C} x_{i}\right)\right)$.

We recall that if $G$ is cyclic then $G_{0}(S[G])$ is isomorphic to the group ring $\mathrm{Z}\left[G^{*}\right]$. Moreover $G^{*} \cong G$. Using this isomorphism as identification and denoting by $\Delta$ the augmentation ideal of $\mathrm{Z}[G], \Delta$ can be identified with the kernel of the map from $G_{0}(S[G])$ to $\mathrm{Z}$, given by $[M] \rightarrow \operatorname{rank}_{S}(M)$ for a $S[G]$-module $M$.

Putting together these observations, we have the following proposition.

Proposition 1.6. Let $G$ be a cyclic group acting on a three dimensional vector space $V$ and let $\left\{\underline{q}_{i}\right\}$ be the set of ramified prime ideals in $\mathrm{C}\left[\left[x_{1}, x_{2}, x_{3}\right]\right]^{G}$. 
Then the following are equivalent:

(1) $\operatorname{Ker} \psi$ is an ideal.

(2) $\operatorname{Ker} \psi$ is the ideal generated by $\left[S / \underline{q}_{i}\right]$.

(3) $\operatorname{Ker} \psi=\Delta^{3}$ or $\Delta^{2}$. Moreover if $\operatorname{Ker} \psi=\Delta^{3}$ then the action is free.

(4) Every Cohen-Macaulay module is a Grothendieck module.

(5) The action is free or $G_{0}(R) \cong \mathrm{Z} \amalg \mathrm{Cl}(R)$.

\section{REFERENCES}

1. M. Auslander, On the purity of the branch locus, Amer. J. Math. 84 (1962), 116-125.

2. _ _ Notes of a course in singularities, Brandeis University, 1985.

3. __ Notes of a course in representation of Artin algebras, Brandeis University.

4. M. Auslander and I. Reiten, Grothendieck groups of algebras and orders, J. Pure Appl. Algebra 39 (1986), 1-51.

5. __ Grothendieck groups with nilpotent annihilators, Proc. Amer. Math. Soc. 103 (1988), 1022-1024.

6. H. Bass, Algebraic K-theory, Benjamin, 1968.

7. S. Bochner and W. T. Martin, Several complex variables, Princeton Univ. Press, 1948.

8. N. Bourbaki, Elements of mathematics, Commutative Algebra, Addison-Wesley, 1972.

9. E. Brieskorn, Rationale Singularitäten Komplexer Flächen, Invent. Math. 4 (1968), 336358.

10. H. Cartan and S. Eilenberg, Homological algebra, Princeton Univ. Press, 1956.

11. R. M. Fossum, The divisor class group of a Krull domain, Springer-Verlag, 1960.

12. N. Jacobson, Basic algebra, Freeman, 1985.

13. M. Nagata, Local rings, Krieger, 1975.

14. P. Samuel and O. Zariski, Commutative algebra, vol. II, Springer-Verlag, 1960.

15. J. P. Serre, Groupes finis d'automorphismes d'anneaux, locaux reguliers, Colloq. d'Algèbre No. 8, École Norm. Sup. de Jeunes Filles, Paris, 1967.

16. R. G. Swan, K-theory of finite groups and orders, Lecture Notes in Math., vol. 149, Springer, 1970.

17. J. A. Wolf, Spaces of constant curvature, McGraw-Hill, 1967.

Departamento de Matemática, Universidade de São Paulo, São Paulo, Brasil

E-mail address: enmarcos@ime.usp.br 